\title{
ALMOST FINITENESS AND THE SMALL BOUNDARY PROPERTY
}

\author{
DAVID KERR AND GÁBOR SZABÓ
}

\begin{abstract}
Working within the framework of free actions of countable amenable groups on compact metrizable spaces, we show that the small boundary property is equivalent to a density version of almost finiteness, which we call almost finiteness in measure, and that under this hypothesis the properties of almost finiteness, comparison, and $m$-comparison for some nonnegative integer $m$ are all equivalent. The proof combines an Ornstein-Weiss tiling argument with the use of zero-dimensional extensions which are measure-isomorphic over singleton fibres. These kinds of extensions are also employed to show that if every free action of a given group on a zero-dimensional space is almost finite then so are all free actions of the group on spaces with finite covering dimension. Combined with recent results of Downarowicz-Zhang and Conley-Jackson-Marks-Seward-Tucker-Drob on dynamical tilings and of Castillejos-Evington-Tikuisis-White-Winter on the Toms-Winter conjecture, this implies that crossed product $\mathrm{C}^{*}$-algebras arising from free minimal actions of groups with local subexponential growth on finite-dimensional spaces are classifiable in the sense of Elliott's program. We show furthermore that, for free actions of countably infinite amenable groups, the small boundary property implies that the crossed product has uniform property $\Gamma$, which confirms the Toms-Winter conjecture for such crossed products in the minimal case.
\end{abstract}

\section{Contents}

1. Introduction 1

2. General terminology and notation 5

3. Almost finiteness in measure and a topological Ornstein-Weiss tower theorem 6

4. Extensions measure-isomorphic over singleton fibres 11

5. Equivalence of the small boundary property and almost finiteness in measure 13

6. Comparison and almost finiteness 18

7. Almost finiteness and the topological small boundary property 19

8. Applications to classifiability 22

9. Crossed products and the Toms-Winter conjecture 25

References 30

\section{INTRODUCTION}

A fundamental feature of the theory of amenability for groups is the connection it establishes between approximate invariance and tiling properties. This connection was made clear in the work of Ornstein and Weiss [37], who showed that the approximate invariance that characterizes amenability via the Følner property can be leveraged through a recursive

Date: May 30, 2019. 
procedure to produce tilings with approximately invariant shapes and almost full coverage, not only within the group itself but also in its probability-measure-preserving actions. In the latter dynamical setting, these tilings (or tower decompositions, to use the more customary terminology for actions) can be translated in a direct way into matrix subalgebras of the von Neumann algebra crossed product and thereby be used to produce a simple proof of hyperfiniteness when the action is free. In particular, this shows that all free ergodic measurepreserving actions of countably infinite amenable groups on atomless standard probability spaces give rise to the unique hyperfinite $\mathrm{II}_{1}$ factor. ${ }^{1}$

The attempt to similarly determine the structure of the $\mathrm{C}^{*}$-crossed products arising from actions of countably infinite amenable groups on compact metrizable spaces has, like the general Elliott classification program for simple separable nuclear $\mathrm{C}^{*}$-algebras, been forced to contend with obstructions of a topological nature that are conditioned by the phenomenon of higher-dimensionality. Although the precise technical connections are still not so well understood, these obstructions at the $\mathrm{C}^{*}$-algebra level are closely related to Gromov's notion of mean dimension [18], which is an entropy-like invariant in topological dynamics that provides a measure of asymptotic dimension growth. Giol and the first author showed in [14] that the cubical subshifts constructed by Lindenstrauss and Weiss in [33] as examples of minimal actions that have nonzero mean dimension also produce crossed products which, for similar structural reasons, fail to behave well from the purview of Elliott's program.

Despite the complications in the $\mathrm{C}^{*}$-picture caused by such obstacles, it has become apparent over the last several years that, from the viewpoint of both structure and classification theory, hyperfiniteness does have a veritable analogue in the $\mathrm{C}^{*}$-realm, namely the conjunction of nuclearity and $\mathscr{Z}$-stability. For a simple separable unital nuclear $\mathrm{C}^{*}$-algebra $A$, the property of $\mathscr{Z}$-stability can be expressed as the existence of order-zero completely positive contractive maps $\varphi$ from matrix algebras into $A$ such that the image of $\varphi$ is approximately central and $1-\varphi(1)$ is small in the sense of Cuntz subequivalence [23]. ${ }^{2}$ The most recent affirmation of this analogy is the work of Castillejos, Evington, Tikuisis, White, and Winter on the Toms-Winter conjecture [4], which shows that $\mathscr{Z}$-stability can be substituted for finite nuclear dimension as the regularity hypothesis in the $\mathrm{C}^{*}$-counterpart to the classification of hyperfinite von Neumann algebras, which itself was only recently clinched in $[12,15,43]$ after several decades of effort by many researchers (see Corollary D and the accompanying discussion in [43]).

This parallel between the measure-theoretic and topological worlds passes moreover to the dynamical level: in [25] it was shown that a topological version of the Ornstein-Weiss tower decomposition called almost finiteness plays the role of $\mathscr{Z}$-stability in a dynamical version of the Toms-Winter conjecture and, when the action is free and minimal, implies that the crossed product is $\mathscr{Z}$-stable. ${ }^{3}$ The main difference between almost finiteness and the Ornstein-Weiss decomposition is that the smallness of the remainder in the former is expressed using dynamical subequivalence instead of a probability measure. While towers

\footnotetext{
${ }^{1}$ The first proof of this fact was given by Connes as an application of his celebrated result [7] that injectivity implies hyperfiniteness, whose full force is still needed to prove hyperfiniteness of the group von Neumann algebra itself.

${ }^{2}$ Whether or not this subequivalence is itself implemented in an approximately central way is roughly what separates $\mathscr{Z}$-stability from its specialization to the nuclear setting.

${ }^{3}$ Nuclearity is automatic in this case since the acting group is amenable.
} 
and tower decompositions have long played an important role in unraveling the structure of $\mathrm{C}^{*}$-crossed products, notably in the case of $\mathbb{Z}$-actions $[39,31,30,44,13]$ and in the various theories of dimension for group actions $[19,41,42,25]$, the novelty in the concept of almost finiteness is its dovetailing of topology with measure-theoretic phenomena within the general setting of amenability. Indeed almost finiteness not only relates to the Ornstein-Weiss tower theorem via analogy but also naturally calls for the direct application of Ornstein-Weiss tiling methods towards $\mathrm{C}^{*}$-algebraic ends, as illustrated by the proof of $\mathscr{Z}$-stability in the free minimal case [25]. This commingling of the measure-theoretic with the topological is very much in line with the kind of $\mathrm{C}^{*}$-application of von Neumann algebra technology that was pioneered by Matui and Sato in their groundbreaking work on the Toms-Winter conjecture $[35,36]$.

One of the main aims of the present paper is to push this technical connection between measure and topology further at the dynamical level. Our work begins with the observation (Theorem 3.13) that the Ornstein-Weiss tiling argument, as presented in [6], applies equally well to free actions of countable amenable groups on zero-dimensional spaces so as to produce a disjoint collection of towers with clopen levels and Følner shapes such that the part of the space that remains uncovered is small in upper density (or, equivalently, uniformly small on all invariant Borel probability measures). This motivates the concept of almost finiteness in measure for free actions on general compact metrizable spaces, which asks for the same kind of tower decomposition with remainder of small upper density but only requires the levels to be open, as is natural for the purpose of accommodating spaces of higher dimension (Definition 3.5). Almost finiteness in measure differs from almost finiteness in replacing the sense in which the remainder is required to be small, from a topological one based on dynamical subequivalence to a measure-theoretic one based on density. In this respect it is similar to the topological Rokhlin property for $\mathbb{Z}^{d}$-actions introduced by Gutman in [20].

By leveraging the above topological version of the Ornstein-Weiss tower theorem via the use of extensions which are measure-isomorphic over singleton fibres, whose basic theory is developed in Section 4, we prove in Theorem 5.6 that, for free actions on compact metrizable spaces, almost finiteness in measure is equivalent to the small boundary property, i.e., the existence of a basis of open sets whose boundaries are null for every invariant Borel probability measure. The small boundary property has its origins in work of Shub and Weiss that initiated an investigation into the problem of when a given dynamical system possesses factors with lower entropy [40]. It is known to imply mean dimension zero, and is conjecturally equivalent to it under freeness, as well as to the decomposability of the action into an inverse limit of finite entropy actions. The equivalence of these three properties for extensions of minimal $\mathbb{Z}$-actions was established in $[33,32]$ and was recently shown in [21] to hold for $\mathbb{Z}$ actions with the marker property and more generally in $[22]$ for $\mathbb{Z}^{d}$-actions with the marker property. As a consequence of Theorem 5.6, we obtain the following as our first main result:

Theorem A (Theorem 6.1). Let $G$ be a countably infinite amenable group. For a free action $G \curvearrowright X$, the following are equivalent:

(i) the action is almost finite,

(ii) the action has the small boundary property and comparison,

(iii) the action has the small boundary property and $m$-comparison for some $m \geq 0$. 
This yields the corollary that, for free minimal actions on compact metrizable spaces of finite covering dimension, finite tower dimension implies almost finiteness (Corollary 6.2).

The type of extensions employed in the proof of Theorem 6.1 are similarly mobilized to show the following reduction theorem.

Theorem B (Corollary 7.7). Let $G$ be a countably infinite amenable group. Suppose that every free action of $G$ on a zero-dimensional compact metrizable space is almost finite. Then it follows that every free action of $G$ on a finite-dimensional compact metrizable space is almost finite. ${ }^{4}$

Recently Downarowicz and Zhang have verified the hypothesis of Theorem B for groups $G$ whose finitely generated subgroups all have subexponential growth [11]. Conley, Jackson, Marks, Seward, and Tucker-Drob also independently established this fact, in unpublished work, by observing that it follows from a clopen version of the tiling argument in [6] (see the introduction to Section 8). Together with the $\mathscr{Z}$-stability theorem from [25], work of Castillejos-Evington-Tikuisis-White-Winter [4], and the classification results from [12, 15, 43], this allows us to deduce our main result about $\mathrm{C}^{*}$-crossed products:

Theorem C (Theorems 8.1 and 8.2). Let $G$ be a countably infinite group for which all finitely generated subgroups have subexponential growth. Then every free action $G \curvearrowright X$ on a compact metrizable finite-dimensional space is almost finite. As a consequence, the crossed products arising from minimal such actions are classified by the Elliott invariant (ordered $K$-theory paired with tracial states) and are simple ASH algebras of topological dimension at most 2 .

This classification theorem was known in the case of finitely generated groups $G$ having polynomial growth (which is equivalent to virtual nilpotence by Gromov's theorem [17]) as a consequence of $[42,1]$, in which it was verified directly that the associated crossed products have finite nuclear dimension. In the polynomial growth setting one can also give a simpler proof of almost finiteness, and hence of $\mathscr{Z}$-stability, as we explain in Section 8 . The more general hypothesis of subexponential growth encompasses groups of intermediate growth such as the Grigorchuk group [16].

A companion paper to [4] shows that the Toms-Winter conjecture holds under the additional hypothesis that the $\mathrm{C}^{*}$-algebra satisfies a $\mathrm{C}^{*}$-version of Murray and von Neumann's property $\Gamma$, called uniform property $\Gamma$ [5]. That is, for nonelementary simple separable unital nuclear $\mathrm{C}^{*}$-algebras satisfying uniform property $\Gamma$, the properties of finite nuclear dimension, $\mathscr{Z}$-stability, and strict comparison are equivalent. Since $\mathscr{Z}$-stability implies uniform property $\Gamma$, this yields the implication from $\mathscr{Z}$-stability to finite nuclear dimension (the reverse implication had been previously established by Winter in [46]). To round out the paper we prove the following about $\mathrm{C}^{*}$-crossed products within the general context of amenable acting groups:

Theorem D (Theorem 9.4 and Corollary 9.5). Let $G$ be a countably infinite amenable group. Let $X$ be a compact metrizable space and $G \curvearrowright X$ a free action with the small boundary property. Then the crossed product $C(X) \rtimes G$ has uniform property $\Gamma$. In particular, the Toms-Winter conjecture holds for such crossed products when the action is also minimal.

\footnotetext{
${ }^{4}$ In fact, this is more generally proved for actions satisfying the so-called topological small boundary property, which is known to be automatic for free actions on finite-dimensional spaces by Theorem 3.8 of [41].
} 
Our expectation is that the conditions in the Toms-Winter conjecture (i.e., finite nuclear dimension, $\mathscr{Z}$-stability, and strict comparison) are in fact automatic for crossed products of free minimal actions of countably infinite amenable groups under the assumption of the small boundary property. This is indeed what happens for $G=\mathbb{Z}$ by a theorem of Elliott and Niu [13]. Their argument relies crucially on the recursive topological structure one obtains by generating tower decompositions via recurrence, which is only available for $\mathbb{Z}$, and so it appears that new methods will be required to tackle the case of $\mathbb{Z}^{2}$ and other amenable groups.

Acknowledgements. The first author was partially supported by NSF grant DMS-1500593. A portion of the work was carried out during his six-month stay in 2017-2018 at the ENS de Lyon, during which time he held ENS and CNRS visiting professorships and was supported by Labex MILYON/ANR-10-LABX-0070. He thanks Damien Gaboriau and Mikael de la Salle at the ENS for their generous hospitality. The second author was supported by EPSRC grant EP/N00874X/1, the Danish National Research Foundation through the Centre for Symmetry and Deformation (DNRF92), the European Union's Horizon 2020 research and innovation programme under the grant MSCA-IF-2016-746272-SCCD, and a start-up grant of KU Leuven. Part of the work was carried out during the second author's visit to Texas A\&M in June 2017, and he thanks the first author for his hospitality. Both authors would like to thank Jianchao Wu for stimulating discussions on the subject of this paper.

\section{General terminology and notation}

Throughout the paper $G$ denotes a countable group. We are mainly interested in the case when $G$ is amenable and will make this a blanket assumption in Section 3 while explicitly inserting it as a hypothesis elsewhere when appropriate.

Given a finite set $K \subseteq G$ and a $\delta>0$, we say that a finite set $F \subseteq G$ is $(K, \delta)$-invariant if $|K F \Delta F|<\delta|F|$. The existence of $(K, \delta)$-invariant finite sets for every finite $K \subseteq G$ and $\delta>0$ is the Følner characterization of amenability for $G$. The notions of upper and lower (Banach) density for a subset $A$ of an amenable $G$, written $\bar{D}(A)$ and $\underline{D}(A)$, are reviewed at the beginning of the next section.

For a compact metrizable space $X$ we write $M(X)$ for the set of Borel probability measures on $X$ equipped with the weak* topology, under which it is compact.

A group action $G \curvearrowright X$ will be expressed using the concatenation $(s, x) \mapsto s x$ except in some instances involving extensions where it will be convenient to give it a name, such as $\alpha$, with the transformation $x \mapsto s x$ of $X$ for a given $s \in X$ sometimes written $\alpha_{s}$.

Actions on compact metrizable spaces are always assumed to be continuous. For an action $G \curvearrowright X$ on a compact metrizable space we write $M_{G}(X)$ for the weak* closed subset of $M(X)$ consisting of all $G$-invariant Borel probability measures on $X$.

Let $\alpha: G \curvearrowright Z$ and $\beta: G \curvearrowright X$ be actions of $G$ on compact metrizable spaces. A map $\pi: Z \rightarrow X$ is a factor map if it is continuous and surjective and satisfies $\pi(s z)=s \pi(x)$ for all $s \in G$ and $z \in Z$ (equivariance). We sometimes write $\pi:(Z, \alpha) \rightarrow(X, \beta)$ to indicate the precise roles of the actions. The map $\pi$ is also called an extension. One also refers to $\beta$ as a factor of $\alpha$ and to $\alpha$ as an extension of $\beta$.

For the remainder of this section we let $G \curvearrowright X$ be a free action on a compact metrizable space. A tower is a pair $(V, S)$ consisting of a subset $V$ of $X$ and a finite subset $S$ of $G$ such 
that the sets $s V$ for $s \in S$ are pairwise disjoint. The set $V$ is the base of the tower, the set $S$ is the shape of the tower, and the sets $s V$ for $s \in S$ are the levels of the tower. The tower $(V, S)$ is open if $V$ is open and clopen if $V$ is clopen. A castle is a finite collection of towers $\left\{\left(V_{i}, S_{i}\right)\right\}_{i \in I}$ such that the sets $S_{i} V_{i}$ for $i \in I$ are pairwise disjoint. The remainder of the castle is the set $X \backslash \bigcup_{i \in I} S_{i} V_{i}$. The castle is open if each of the towers is open, and clopen if each of the towers is clopen.

Let $m \geq 0$ and $A, B \subseteq X$. Write $A \prec_{m} B$ if for every closed set $C \subseteq A$ there is a finite collection $\mathscr{U}$ of open subsets of $X$ which cover $C$, an $s_{U} \in G$ for each $U \in \mathscr{U}$, and a partition $\mathscr{U}=\mathscr{U}_{0} \sqcup \cdots \sqcup \mathscr{U}_{m}$ such that for each $i=0, \ldots, m$ the sets $s_{U} U$ for $U \in \mathscr{U}_{i}$ are pairwise disjoint and contained in $B$. When $m=0$ we also write $A \prec B$, in which case we say that $A$ is subequivalent to $B$. The action $G \curvearrowright X$ has $m$-comparison if $A \prec_{m} B$ for all nonempty open sets $A, B \subseteq X$ satisfying $\mu(A)<\mu(B)$ for every $\mu \in M_{G}(X)$. In the case $m=0$ we also simply say that the action has comparison.

The action $G \curvearrowright X$ is said to be almost finite if for every $n \in \mathbb{N}$, finite set $K \subseteq G$, and $\delta>0$ there are

(i) an open castle $\left\{\left(V_{i}, S_{i}\right)\right\}_{i \in I}$ whose shapes are $(K, \delta)$-invariant and whose levels have diameter less than $\delta$,

(ii) sets $S_{i}^{\prime} \subseteq S_{i}$ such that $\left|S_{i}^{\prime}\right|<\left|S_{i}\right| / n$ and

$$
X \backslash \bigsqcup_{i \in I} S_{i} V_{i} \prec \bigsqcup_{i \in I} S_{i}^{\prime} V_{i}
$$

When $X$ is zero-dimensional this agrees with Matui's original notion of almost finiteness for second countable étale groupoids with compact zero-dimensional unit spaces [34]. In this case one can equivalently require that the levels of the tower be clopen and partition $X$, so that condition (ii) can be dispensed with. See Section 10 of [25].

\section{Almost finiteness in measure and a topological Ornstein-Weiss tower THEOREM}

The proof of the Ornstein-Weiss tower theorem as presented in [6] shows, by substituting clopenness for measurability, that every free action of an amenable group on a zerodimensional compact metrizable space is almost finite in measure (Definition 3.5). For the convenience of the reader we will briefly reconstruct the argument as it applies to establish the clopen version, which we record as Theorem 3.13. Along the way we will also establish a couple of facts (Propositions 3.3 and 3.4) that will be useful in later sections.

Throughout the section we assume $G$ to be amenable.

Let $G \curvearrowright X$ be an arbitrary action on a set.

Definition 3.1. For a set $A \subseteq X$ we define

$$
\underline{D}(A)=\sup _{F} \inf _{x \in X} \frac{1}{|F|} \sum_{s \in F} 1_{A}(s x) \quad \text { and } \quad \bar{D}(A)=\inf _{F} \sup _{x \in X} \frac{1}{|F|} \sum_{s \in F} 1_{A}(s x)
$$

where $F$ ranges over the nonempty finite subsets of $G$ in both cases. These two quantities are called the lower and upper (Banach) densities of $A$.

Observe that $\underline{D}(A)=1-\bar{D}(X \backslash A)$ and $\underline{D}(s A)=\underline{D}(A)$ for all $s \in G$. 
The following is a dynamical version of Lemma 2.9 in [10] and can be established by the same argument.

Lemma 3.2. Let $\left(F_{n}\right)_{n}$ be a Følner sequence for $G$. Then

$$
\underline{D}(A)=\lim _{n \rightarrow \infty} \inf _{x \in X} \frac{1}{\left|F_{n}\right|} \sum_{s \in F_{n}} 1_{A}(s x) \quad \text { and } \quad \bar{D}(A)=\lim _{n \rightarrow \infty} \sup _{x \in X} \frac{1}{\left|F_{n}\right|} \sum_{s \in F_{n}} 1_{A}(s x) .
$$

Proposition 3.3. Let $G \curvearrowright X$ be a continuous action on a compact metrizable space and $A$ a subset of $X$. If $A$ is closed, then

$$
\bar{D}(A)=\sup _{\mu \in M_{G}(X)} \mu(A)
$$

while if $A$ is open then

$$
\underline{D}(A)=\inf _{\mu \in M_{G}(X)} \mu(A) .
$$

Proof. By the observation following Definition 3.1, we need only prove the first assertion. Suppose then that $A$ is closed. By Lemma 3.2 there are a Følner sequence $\left(F_{n}\right)_{n}$ for $G$ and a sequence $\left\{x_{n}\right\}$ in $X$ such that

$$
\bar{D}(A)=\lim _{n \rightarrow \infty} \frac{1}{\left|F_{n}\right|} \sum_{t \in F_{n}} 1_{A}\left(t x_{n}\right) .
$$

Take a weak* cluster point $\mu$ of the sequence $\mu_{n}:=\left|F_{n}\right|^{-1} \sum_{t \in F_{n}} \delta_{t x_{n}}$ in $M(X)$, where $\delta_{t x_{n}}$ denotes the point mass at $t x_{n}$. Then for every $f \in C(X)$ and $s \in G$ we have, writing $s f$ for the function $x \mapsto f\left(s^{-1} x\right)$ and abbreviating integration with respect to $\mu$ and $\mu_{n}$ as $\mu(\cdot)$ and $\mu_{n}(\cdot)$

$$
\begin{aligned}
|\mu(s f)-\mu(f)| & \leq\left|\mu(s f)-\mu_{n}(s f)\right|+\left|\mu_{n}(s f)-\mu_{n}(f)\right|+\left|\mu_{n}(f)-\mu(f)\right| \\
& \leq\left|\mu(s f)-\mu_{n}(s f)\right|+\frac{\left|s^{-1} F_{n} \Delta F_{n}\right|}{\left|F_{n}\right|} \| f||+\left|\mu_{n}(f)-\mu(f)\right| \\
& \rightarrow 0
\end{aligned}
$$

as $n \rightarrow \infty$, showing that $\mu$ is $G$-invariant. Moreover, the portmanteau theorem yields

$$
\mu(A) \geq \limsup _{n \rightarrow \infty} \mu_{n}(A)=\lim _{n \rightarrow \infty} \frac{1}{\left|F_{n}\right|} \sum_{t \in F_{n}} 1_{A}\left(t x_{n}\right)=\bar{D}(A)
$$

and hence $\bar{D}(A) \leq \sup _{\mu \in M_{G}(X)} \mu(A)$.

For the reverse inequality, it suffices to observe that for every $\mu \in M_{G}(X)$ and nonempty finite set $F \subseteq G$ we have

$$
\begin{aligned}
\mu(A)=\frac{1}{|F|} \sum_{t \in F} \int_{X} 1_{t^{-1} A} d \mu & =\int_{X} \frac{1}{|F|} \sum_{t \in F} 1_{t^{-1} A} d \mu \\
& \leq\left\|\frac{1}{|F|} \sum_{t \in F} 1_{t^{-1} A}\right\|=\sup _{x \in X} \frac{1}{|F|} \sum_{t \in F} 1_{A}(t x) .
\end{aligned}
$$

so that $\mu(A) \leq \bar{D}(A)$. 
Proposition 3.4. Let $X$ be a compact metrizable space with compatible metric $d$ and let $A$ be a closed subset of $X$. Then for every $\varepsilon>0$ there exists a $\delta>0$ such that the set

$$
A_{+}=\{x \in X: d(x, A) \leq \delta\}
$$

satisfies $\bar{D}\left(A_{+}\right) \leq \bar{D}(A)+\varepsilon$.

Proof. Suppose to the contrary that there is an $\varepsilon>0$ for which no suitable $\delta$ exists. Then by Proposition 3.3 we can find, for every $n \in \mathbb{N}$, a $\mu_{n} \in M_{G}(X)$ such that the set

$$
A_{n}=\{x \in X: d(x, A) \leq 1 / n\}
$$

satisfies $\mu_{n}\left(A_{n}\right)>\bar{D}(A)+\varepsilon / 2$. Since $M_{G}(X)$ is compact in the weak* topology there is a subsequence $\left(\mu_{n_{k}}\right)_{k}$ of $\left(\mu_{n}\right)_{n}$ which weak* converges to some $\mu \in M_{G}(X)$. For integers $k \geq j \geq 1$ we have

$$
\mu_{n_{k}}\left(A_{n_{j}}\right) \geq \mu_{n_{k}}\left(A_{n_{k}}\right)>\bar{D}(A)+\varepsilon / 2,
$$

and so for a fixed $j$ the portmanteau theorem yields, since $A_{n_{j}}$ is closed,

$$
\mu\left(A_{n_{j}}\right) \geq \limsup _{k \rightarrow \infty} \mu_{n_{k}}\left(A_{n_{j}}\right) \geq \bar{D}(A)+\varepsilon / 2 .
$$

Since $A$ is equal to the decreasing intersection of the sets $A_{n_{j}}$ for $j \in \mathbb{N}$, we deduce that

$$
\mu(A)=\lim _{j \rightarrow \infty} \mu\left(A_{n_{j}}\right) \geq \bar{D}(A)+\varepsilon / 2 .
$$

On the other hand $\mu(A) \leq \bar{D}(A)$ by Proposition 3.3, producing a contradiction.

For the rest of this section we will assume that the action $G \curvearrowright X$ is free. Under this assumption, we can write the sum appearing in Definition 3.1 as a cardinality via the equation

$$
\sum_{t \in F} 1_{A}(t x)=|A \cap F x|
$$

for all $x \in X, A \subseteq X$ and finite subsets $F \subseteq G$.

Definition 3.5. A free action $G \curvearrowright X$ on a compact metric space is said to be almost finite in measure if for every finite set $K \subseteq G$ and $\delta, \varepsilon>0$ there is an open castle $\left\{\left(V_{i}, S_{i}\right)\right\}_{i \in I}$ with levels of diameter less than $\delta$ such that

(i) each shape $S_{i}$ is $(K, \delta)$-invariant,

(ii) $\underline{D}\left(\bigsqcup_{i \in I} S_{i} V_{i}\right) \geq 1-\varepsilon$.

Definition 3.6. Let $K$ be a finite subset of $G$ and $\delta>0$. A set $A \subseteq X$ is said to be $(K, \delta)^{*}$-invariant if there exists a finite set $F \subseteq G$ such that

$$
|(K A \Delta A) \cap F x|<\delta|A \cap F x|
$$

for all $x \in X$.

Definition 3.7. Let $\varepsilon>0$. A collection $\left\{A_{i}\right\}_{i \in I}$ of finite subsets of $G$ is said to be $\varepsilon$-disjoint if for every $i \in I$ there is a subset $A_{i}^{\prime} \subseteq A_{i}$ with $\left|A_{i}^{\prime}\right| \geq(1-\varepsilon)\left|A_{i}\right|$ such that the sets $A_{i}^{\prime}$ for $i \in I$ are pairwise disjoint.

One can establish the following in the same way as Lemma 3.2. 
Lemma 3.8. Let $K$ be a finite subset of $G$ and $\delta>0$. Let $\left(F_{n}\right)_{n}$ be a Følner sequence for $G$. Then a set $A \subseteq X$ is $(K, \delta)^{*}$-invariant if and only if $A \cap F_{n} x \neq \emptyset$ for all $x \in X$ and sufficiently large $n$ and

$$
\lim _{n \rightarrow \infty} \sup _{x \in X} \frac{\left|(K A \Delta A) \cap F_{n} x\right|}{\left|A \cap F_{n} x\right|}<\delta .
$$

The following is Lemma 3.1 from [6].

Lemma 3.9. Let $K$ and $F$ be finite subsets of $G$ and let $\varepsilon, \delta>0$. Let $C$ be a subset of $X$ and for each $c \in C$ let $F_{c}$ be $a(K, \delta(1-\varepsilon))$-invariant subset of $F$ so that the collection $\left\{F_{c} c: c \in C\right\}$ is $\varepsilon$-disjoint and $\underline{D}\left(\bigcup_{c \in C} F_{c} c\right)>0$. Then $\bigcup_{c \in C} F_{c} c$ is $(K, \delta)^{*}$-invariant.

The following is Lemma 3.2 from [6].

Lemma 3.10. Let $T$ be a finite subset of $G$ and let $\varepsilon, \delta>0$ be such that $\varepsilon(1+\delta)<1$. Let $B$ be $a\left(T^{-1}, \delta\right)^{*}$-invariant subset of $X$, and let $A$ be a subset of $X$ with $B \subseteq A$ such that $|A \cap T x| \geq \varepsilon|T|$ for all $x \in X$. Then

$$
\underline{D}(A) \geq(1-\varepsilon(1+\delta)) \underline{D}(B)+\varepsilon .
$$

Lemma 3.11. Let $X$ be a zero-dimensional compact metrizable space and $G \curvearrowright X$ a free action. Let $Y$ be a clopen subset of $X$ and $S$ a finite subset of $G$. Let $0<\varepsilon<\frac{1}{2}$ and $\delta>0$. Then there is a clopen castle $\left\{\left(V_{i}, S_{i}\right)\right\}_{i=1}^{n}$ with levels of diameter less than $\delta$ such that

(i) $S_{i} \subseteq S$ and $\left|S_{i}\right| \geq(1-\varepsilon)|S|$ for every $i=1, \ldots, n$,

(ii) the set $A=\bigsqcup_{i=1}^{n} S_{i} V_{i}$ satisfies $Y \cap A=\emptyset, Y \cup A=Y \cup \bigcup_{i=1}^{n} S V_{i}$, and $|Y \cup A \cap S x| \geq$ $\varepsilon|S|$ for all $x \in X$.

Proof. By freeness and zero-dimensionality we can find a clopen partition $\left\{V_{1}, \ldots, V_{m}\right\}$ of $X$ such that for each $i=1, \ldots, m$ the pair $\left(V_{i}, S\right)$ is a tower whose levels all have diameter less than $\delta$. Write $\mathscr{T}$ for the set of all $T \subseteq S$ such that $|T| \geq(1-\varepsilon)|S|$. Set $A_{0}=Y$. We will recursively construct sets $A_{1}, \ldots, A_{m}$ and clopen castles $\left\{\left(V_{i, T}, T\right)\right\}_{T \in \mathscr{T}}$ for $i=1, \ldots, m$, with some of the sets $V_{i, T}$ possibly being empty, such that $A_{i}$ is the disjoint union of $A_{i-1}$ and $\bigsqcup_{T \in \mathscr{T}} T V_{i, T}$ for each $i=1, \ldots, m$.

Let $1 \leq i \leq m$ and suppose that we have constructed the sets $A_{1}, \ldots, A_{i-1}$ and (in the case $i>1$ ) clopen castles $\left\{\left(V_{j, T}, T\right)\right\}_{T \in \mathscr{T}}$ for $j=1, \ldots, i-1$ satisfying the required properties. For each $T \in \mathscr{T}$ define the clopen set

$$
V_{i, T}=V_{i} \cap\left(\bigcap_{s \in S \backslash T} s^{-1} A_{i-1}\right) \cap\left(\bigcap_{s \in T}\left(X \backslash s^{-1} A_{i-1}\right)\right) .
$$

The sets $V_{i, T}$ are pairwise disjoint, and so $\left\{\left(V_{i, T}, T\right)\right\}_{T \in \mathscr{T}}$ is a clopen castle such that $A_{i-1}$ and $\bigsqcup_{T \in \mathscr{T}} T V_{i, T}$ are disjoint. Put $A_{i}=A_{i-1} \bigsqcup_{T \in \mathscr{T}} T V_{i, T}$ to complete the recursive construction.

Set $A=\bigsqcup_{i=1}^{m} \bigsqcup_{T \in \mathscr{T}} T V_{i, T}$. Then $Y \cap A=\emptyset$ and $Y \cup A=Y \cup \bigcup_{i=1}^{m} \bigcup_{T \in \mathscr{T}} S V_{i, T}$. Let $x \in X$. Then there is an $1 \leq i \leq m$ such that $x \in V_{i}$. If $x \in V_{i, T}$ for some $T \in \mathscr{T}$ then $T x \subseteq A_{i} \subseteq Y \cup A$ and hence

$$
|Y \cup A \cap S x| \geq|T x|=|T| \geq(1-\varepsilon)|S| \geq \varepsilon|S| .
$$

On the other hand, if $x \notin V_{i, T}$ for all $T \in \mathscr{T}$ then the set of all $s \in S$ such that $s x \in A_{i}$ has cardinality at least $\varepsilon|S|$ and hence $|Y \cup A \cap S x| \geq\left|A_{i} \cap S x\right| \geq \varepsilon|S|$. Thus the clopen castle 
$\left\{\left(V_{j, T}, T\right)\right\}_{1 \leq j \leq m, T \in \mathscr{T}}$ satisfies condition (ii) in the lemma statement. Condition (i) is built into the construction.

The following is a simple (and well-known) exercise.

Lemma 3.12. Let $K$ be a finite subset of $G$ and $\delta>0$. Then there is an $\varepsilon>0$ such that if $F$ is a $(K, \varepsilon)$-invariant finite subset of $G$ then every set $F^{\prime} \subseteq F$ with $\left|F^{\prime}\right| \geq(1-\varepsilon)|F|$ is $(K, \delta)$-invariant.

Theorem 3.13. A free action $G \curvearrowright X$ on a zero-dimensional compact metrizable space is almost finite in measure.

Proof. Let $K$ be a finite subset of $G$ and $\delta>0$. Let $\varepsilon>0$ be such that $\varepsilon(1+\delta)<1$ and also such that it satisfies the conclusion of Lemma 3.12 with respect to $K$ and $\delta$. Take an $n \in \mathbb{N}$ such that $(1-\varepsilon)^{n}<\varepsilon$.

Fix a $\beta>0$ such that $(1+\beta)^{-1}\left(1-(1-(1+\beta) \varepsilon)^{n}\right)>1-\varepsilon$ and take nonempty $(K, \varepsilon)$-invariant finite sets $F_{1}, \ldots, F_{n} \subseteq G$ such that for all $1 \leq j<i \leq n$ the set $F_{i}$ is $\left(F_{j}^{-1}, \beta(1-\varepsilon)\right)$-invariant. By a recursive procedure we will construct, for each $i$ running from $n$ down to 1 , a clopen castle $\left\{\left(V_{i, k}, F_{i, k}\right)\right\}_{k=1}^{k_{i}}$ with levels of diameter less than $\delta$ and $F_{i, k} \subseteq F_{i}$ and $\left|F_{i, k}\right| \geq(1-\varepsilon)\left|F_{i}\right|$ for $k=1, \ldots, k_{i}$ so that the sets $A_{i}=\bigsqcup_{k=1}^{k_{i}} F_{i, k} V_{i, k}$ are pairwise disjoint and satisfy

$$
\bigcup_{j=i}^{n} A_{j}=\bigcup_{j=i+1}^{n} A_{j} \cup \bigcup_{k=1}^{k_{i}} F_{i} V_{i, k}
$$

and

$$
\underline{D}\left(A_{i} \cup A_{i+1} \cup \cdots \cup A_{n}\right) \geq \frac{1}{1+\beta}\left(1-(1-\varepsilon(1+\beta))^{n+1-i}\right) .
$$

Since each $F_{i, k}$ will be $(K, \delta)$-invariant by our choice of $\varepsilon$, this will establish the theorem.

For the first stage of the construction, apply Lemma 3.11 with $Y=\emptyset$ and $S=F_{n}$ to obtain a clopen castle $\left\{\left(V_{n, k}, F_{n, k}\right)\right\}_{k=1}^{k_{n}}$ with levels of diameter less than $\delta$ such that

(i) $F_{n, k} \subseteq F_{n}$ and $\left|F_{n, k}\right| \geq(1-\varepsilon)\left|F_{n}\right|$ for every $k=1, \ldots, k_{n}$,

(ii) the set $A_{n}=\bigsqcup_{k=1}^{k_{n}} F_{n, k} V_{n, k}$ satisfies $A_{n}=\bigcup_{k=1}^{k_{n}} F_{n} V_{n, k}$ and $\left|A_{n} \cap F_{n} x\right| \geq \varepsilon\left|F_{n}\right|$ for all $x \in X$.

Apply Lemma 3.10 with $B=\emptyset$ and $A=A_{n}$ to obtain $\underline{D}\left(A_{n}\right) \geq \varepsilon$.

Now let $1 \leq i<n$ and suppose that we have carried out the stages of the construction from $n$ down to $i+1$. Apply Lemma 3.11 with $Y=\bigsqcup_{j=i+1}^{n} A_{j}$ and $S=F_{i}$ to obtain a clopen castle $\left\{\left(V_{i, k}, F_{i, k}\right)\right\}_{k=1}^{k_{i}}$ with levels of diameter less than $\delta$ such that

(i) $F_{i, k} \subseteq F_{i}$ and $\left|F_{i, k}\right| \geq(1-\varepsilon)\left|F_{i}\right|$ for every $k=1, \ldots, k_{i}$,

(ii) the set $A_{i}=\bigsqcup_{k=1}^{k_{i}} F_{i, k} V_{i, k}$ satisfies $A_{i} \cap\left(A_{i+1} \cup \cdots \cup A_{n}\right)=\emptyset, \bigsqcup_{j=i}^{n} A_{j}=\bigsqcup_{j=i+1}^{n} A_{j} \cup$ $\bigcup_{k=1}^{k_{i}} F_{i} V_{i, k}$, and $\left|\bigsqcup_{j=i}^{n} A_{j} \cap F_{i} x\right| \geq \varepsilon\left|F_{i}\right|$ for all $x \in X$.

By Lemma 3.9 the set $\bigsqcup_{j=i+1}^{n} A_{j}$ is $\left(F_{i}^{-1}, \beta\right)^{*}$-invariant and so we can apply Lemma 3.10 with $B=\bigsqcup_{j=i+1}^{n} A_{j}$ and $A=\bigsqcup_{j=i}^{n} A_{j}$ to obtain

$$
\underline{D}\left(A_{i} \cup \cdots \cup A_{n}\right) \geq(1-\varepsilon(1+\beta)) \underline{D}\left(A_{i+1} \cup \cdots \cup A_{n}\right)+\varepsilon
$$




$$
\begin{aligned}
& \geq \frac{1}{1+\beta}\left(1-\varepsilon(1+\beta)-(1-\varepsilon(1+\beta))^{n+1-i}+\varepsilon(1+\beta)\right) \\
& =\frac{1}{1+\beta}\left(1-(1-\varepsilon(1+\beta))^{n+1-i}\right),
\end{aligned}
$$

completing the $i$ th stage of the construction.

\section{EXTENSIONS MEASURE-ISOMORPHIC OVER SINGLETON FIBRES}

The proofs of Theorems 5.5 and 7.3 will hinge on the use of extensions which are measureisomorphic over singleton fibres (Definition 4.2), about which we develop some basic facts here.

Let $Z$ and $X$ be compact metrizable spaces and $\pi: Z \rightarrow X$ a continuous surjection. Write $S_{\pi}$ for the set of all $x \in X$ such that $\pi^{-1}(x)$ is a singleton. Then $S_{\pi}$ is a $G_{\delta}$ set, for if we fix a compatible metric on $X$ then we can write $S_{\pi}$ as the intersection of the open sets $\left\{x \in X: \operatorname{diam}\left(\pi^{-1}(x)\right)<1 / n\right\}$ for $n \in \mathbb{N}$.

Proposition 4.1. For $\mu \in M(Z)$ the following are equivalent:

(i) $\pi_{*} \mu\left(S_{\pi}\right)=1$,

(ii) there is a basis $\mathscr{B}$ for the topology on $Z$ such that for every $U \in \mathscr{B}$ one has $\pi_{*} \mu(\pi(U) \cap \pi(Z \backslash U))=0$,

(iii) for every open set $U \subseteq Z$ one has $\pi_{*} \mu(\pi(U) \cap \pi(Z \backslash U))=0$,

(iv) for every pair of disjoint closed sets $C_{1}, C_{2} \subseteq Z$ one has $\pi_{*} \mu\left(\pi\left(C_{1}\right) \cap \pi\left(C_{2}\right)\right)=0$,

(v) for every closed set $C \subseteq Z$ one has $\pi_{*} \mu(\pi(C))=\mu(C)$,

(vi) for every open set $U \subseteq Z$ one has $\pi_{*} \mu(\pi(U))=\mu(U)$.

Proof. (i) $\Rightarrow$ (vi). For every open set $U \subseteq Z$ we have $\pi^{-1}\left(S_{\pi} \cap \pi(U)\right)=\pi^{-1}\left(S_{\pi}\right) \cap U$ and hence, using (i),

$$
\mu(U) \leq \pi_{*} \mu(\pi(U))=\pi_{*} \mu\left(S_{\pi} \cap \pi(U)\right)=\mu\left(\pi^{-1}\left(S_{\pi}\right) \cap U\right)=\mu(U) .
$$

$(\mathrm{vi}) \Rightarrow(\mathrm{v})$. Let $C$ be a closed subset of $Z$. Take a decreasing sequence $U_{1} \supseteq U_{2} \supseteq \ldots$ of open subsets of $Z$ such that $C=\bigcap_{n=1}^{\infty} U_{n}$. Then $\pi(C)$ is equal to the intersection of the decreasing sequence

$$
\pi\left(U_{1}\right) \supseteq \pi\left(U_{2}\right) \supseteq \ldots
$$

and so (vi) yields

$$
\pi_{*} \mu(\pi(C))=\lim _{n \rightarrow \infty} \pi_{*} \mu\left(\pi\left(U_{n}\right)\right)=\lim _{n \rightarrow \infty} \mu\left(U_{n}\right)=\mu(C) .
$$

(v) $\Rightarrow\left(\right.$ iv). Let $C_{1}$ and $C_{2}$ be disjoint closed subsets of $Z$. Then since $\pi\left(C_{1}\right) \cup \pi\left(C_{2}\right)=$ $\pi\left(C_{1} \cup C_{2}\right)$ we can apply (v) to obtain

$$
\begin{aligned}
\pi_{*} \mu\left(\pi\left(C_{1}\right) \cap \pi\left(C_{2}\right)\right) & =\pi_{*} \mu\left(\pi\left(C_{1}\right)\right)+\pi_{*} \mu\left(\pi\left(C_{2}\right)\right)-\pi_{*} \mu\left(\pi\left(C_{1}\right) \cup \pi\left(C_{2}\right)\right) \\
& =\mu\left(C_{1}\right)+\mu\left(C_{2}\right)-\mu\left(C_{1} \cup C_{2}\right)=0 .
\end{aligned}
$$

(iv) $\Rightarrow$ (iii). Let $U$ be an open subset of $Z$. Take an increasing sequence $C_{1} \subseteq C_{2} \subseteq \ldots$ of closed subsets of $Z$ such that $U=\bigcup_{n=1}^{\infty} C_{n}$. By (iv), for every $n$ we have

$$
\pi_{*} \mu\left(\pi\left(C_{n}\right) \cap \pi(X \backslash U)\right)=0 .
$$


Thus

$$
\begin{aligned}
\pi_{*} \mu(\pi(U) \cap \pi(X \backslash U)) & =\pi_{*} \mu\left(\left(\bigcup_{n=1}^{\infty} \pi\left(C_{n}\right)\right) \cap \pi(X \backslash U)\right) \\
& =\pi_{*} \mu\left(\bigcup_{n=1}^{\infty}\left(\pi\left(C_{n}\right) \cap \pi(X \backslash U)\right)\right) \\
& =\lim _{n \rightarrow \infty} \pi_{*} \mu\left(\pi\left(C_{n}\right) \cap \pi(X \backslash U)\right)=0,
\end{aligned}
$$

yielding (iii).

(iii) $\Rightarrow$ (ii). Trivial.

(ii) $\Rightarrow$ (i). Let $\mathscr{B}$ be as in (ii). Fix a compatible metric $d$ on $X$. Given an $n \in \mathbb{N}$, we define the set $W_{n}=\left\{z \in Z: \operatorname{diam}\left(\pi^{-1}(\pi(z))\right) \geq 1 / n\right\}$, which is readily seen to be closed. By compactness we can find open sets $U_{1}, \ldots, U_{k_{n}} \in \mathscr{B}$ of diameter at most $1 /(2 n)$ whose union contains $W_{n}$. Then for each $j=1, \ldots, k_{n}$ we have $\pi\left(U_{j}\right) \cap \pi\left(W_{n}\right) \subseteq \pi\left(Z \backslash U_{j}\right)$, and since by (ii) we have $\pi_{*} \mu\left(\pi\left(U_{j}\right) \cap \pi\left(Z \backslash U_{j}\right)\right)=0$ we deduce that $\pi_{*} \mu\left(\pi\left(U_{j}\right) \cap \pi\left(W_{n}\right)\right)=0$ and hence $\mu\left(U_{j} \cap W_{n}\right)=0$. Therefore

$$
\mu\left(W_{n}\right) \leq \sum_{j=1}^{k_{n}} \mu\left(U_{j} \cap W_{n}\right)=0 .
$$

It follows that $\mu\left(\bigcup_{n=1}^{\infty} W_{n}\right)=\lim _{n \rightarrow \infty} \mu\left(W_{n}\right)=0$. Since $\bigcup_{n=1}^{\infty} W_{n}$ is equal to $Z \backslash \pi^{-1}\left(S_{\pi}\right)$, we conclude that $\pi_{*} \mu\left(S_{\pi}\right)=1$.

Now let $G \curvearrowright Z$ and $G \curvearrowright X$ be actions on compact metrizable spaces and let $\pi: Z \rightarrow X$ be a factor map. Note that the $G_{\delta}$ set $S_{\pi}$ defined at the beginning of the section is $G$-invariant in this case.

Definition 4.2. The extension $\pi$ is measure-isomorphic if for every $\mu \in M_{G}(Z)$ the map $\pi$ induces a measure conjugacy between the measure-preserving actions $G \curvearrowright(Z, \mu)$ and $G \curvearrowright\left(X, \pi_{*} \mu\right)$, i.e., $\pi$ restricts to an equivariant measure isomorphism between some $G$ invariant conull subsets of $Z$ and $X$. The extension $\pi$ is measure-isomorphic over singleton fibres if $\pi_{*} \mu\left(S_{\pi}\right)=1$ for every $\mu \in M_{G}(Z)$.

Note that if $\pi$ is measure-isomorphic over singleton fibres then it is measure-isomorphic, as $\pi$ restricts to a $G$-equivariant Borel isomorphism $\pi^{-1}\left(S_{\pi}\right) \rightarrow S_{\pi}$ ([24], Corollary 15.2). The converse is false, as examples in [9] demonstrate.

The following is immediate from Proposition 4.1.

Proposition 4.3. For the extension $\pi: Z \rightarrow X$ the following are equivalent:

(i) $\pi$ is measure-isomorphic over singleton fibres,

(ii) there is a basis $\mathscr{B}$ for the topology on $Z$ such that for every $U \in \mathscr{B}$ one has $\pi_{*} \mu(\pi(U) \cap \pi(Z \backslash U))=0$ for all $\mu \in M_{G}(Z)$,

(iii) for every open set $U \subseteq Z$ one has $\pi_{*} \mu(\pi(U) \cap \pi(Z \backslash U))=0$ for all $\mu \in M_{G}(Z)$,

(iv) for every pair of disjoint closed sets $C_{1}, C_{2} \subseteq Z$ one has $\pi_{*} \mu\left(\pi\left(C_{1}\right) \cap \pi\left(C_{2}\right)\right)=0$ for all $\mu \in M_{G}(Z)$,

(v) for every closed set $C \subseteq Z$ one has $\pi_{*} \mu(\pi(C))=\mu(C)$ for all $\mu \in M_{G}(Z)$,

(vi) for every open set $U \subseteq Z$ one has $\pi_{*} \mu(\pi(U))=\mu(U)$ for all $\mu \in M_{G}(Z)$. 
Note that if $G$ is amenable then one has $\pi_{*}\left(M_{G}(Z)\right)=M_{G}(X)$ as a consequence of the Hahn-Banach theorem. To be more precise, we can view a given measure $\nu$ in $M_{G}(X)$ as a $G$-invariant state $\sigma$ on the $\mathrm{C}^{*}$-algebra $C(X)$ and, regarding $C(X)$ as a $\mathrm{C}^{*}$-subalgebra of $C(Z)$ under the embedding given by composition with $\pi$, we can extend $\sigma$ by the Hahn-Banach theorem to a state $\tilde{\sigma}$ on $C(Z)$. Using compactness of the state space, we may then take a weak ${ }^{*}$ cluster point of a sequence of averaged states given by $f \mapsto\left|F_{n}\right|^{-1} \sum_{s \in F_{n}} \tilde{\sigma}\left(s^{-1} f\right)$ over a Følner sequence $\left\{F_{n}\right\}$ of $G$, which yields a $G$-invariant state on $C(Z)$ restricting to $\sigma$ on $C(X)$. By the Riesz representation theorem, such a state corresponds to a unique measure in $M_{G}(Z)$ that maps to $\nu$ under (the push-forward of) $\pi$.

In particular, one can replace " $\pi_{*} \mu$ " and "for all $\mu \in M_{G}(Z)$ " with " $\nu$ " and "for all $\nu \in$ $M_{G}(X)$ ", respectively, in each of the conditions (ii), (iii), and (iv) in the above proposition.

\section{Equivalence of the SMALl BOUNDARY PROPERTY AND ALMOST FINITENESS IN} MEASURE

Our goal in this section is to establish Theorem 5.6 equating almost finiteness in measure with the small boundary property under the assumption of freeness. Below $X$ is assumed to be a compact metrizable space.

Let us begin by recalling the small boundary property. This concept originated in the work of Shub and Weiss (Section 1 of [40]) and later appeared in the context of actions with mean dimension zero [33].

Definition 5.1. An action $G \curvearrowright X$ is said to have the small boundary property if there is a basis for the topology on $X$ consisting of open sets $U$ such that $\mu(\partial U)=0$ for every $\mu \in M_{G}(X)$.

When $G$ is amenable, the condition on $U$ above can be rephrased as $\bar{D}(\partial U)=0$ in view of Proposition 3.3.

Recall that a set $C \subseteq X$ is said to be regular closed if $C=\overline{\operatorname{int}(C)}$.

Definition 5.2. A finite collection $\mathscr{P}$ of subsets of $X$ is called a regular closed partition if all of its members are regular closed, $X=\bigcup \mathscr{P}$, and $C_{1} \cap C_{2} \subseteq \partial C_{1} \cap \partial C_{2}$ for all distinct elements $C_{1}, C_{2} \in \mathscr{P}$. In this case we define the boundary of $\mathscr{P}$ by

$$
\partial \mathscr{P}=\bigcup_{C \in \mathscr{P}} \partial C \subseteq X .
$$

If $\alpha$ is some homeomorphism from $X$ to itself, then $\alpha(\mathscr{P})$ will denote the regular closed partition given by the family $\{\alpha(C) \mid C \in \mathscr{P}\}$. In other words, we identify $\alpha$ with its induced bijection on the set of all regular closed partitions on $X$.

A regular closed partition is not a genuine partition in general, but can be viewed as a partition modulo a set which is both closed and nowhere dense and hence topologically small in a manner of speaking.

Remark 5.3. Let $\mathscr{P}_{1}$ and $\mathscr{P}_{2}$ be two regular closed partitions of $X$. As for open covers, we say that $\mathscr{P}_{1}$ refines $\mathscr{P}_{2}$ if there exists a map $r: \mathscr{P}_{1} \rightarrow \mathscr{P}_{2}$ such that $C \subseteq r(C)$ for all $C \in \mathscr{P}_{1}$. We refer to such a map as a refinement map. Unlike for open covers, it follows easily from the definition of a regular closed partition that such a refinement map, if it exists, is unique. 
Definition 5.4. Let $\mathscr{P}_{1}$ and $\mathscr{P}_{2}$ be two regular closed partitions of $X$. We define their common refinement by

$$
\mathscr{P}_{1} \vee \mathscr{P}_{2}=\left\{\overline{\operatorname{int}\left(C_{1} \cap C_{2}\right)} \mid C_{1} \in \mathscr{P}_{1}, C_{2} \in \mathscr{P}_{2}\right\}
$$

which is easily seen to be another regular closed partition.

The main thrust of the following theorem lies in the construction proving the implication (i) $\Rightarrow$ (ii), which draws inspiration from a similar construction in [29] carried out for $G=\mathbb{Z}$ in a slightly different context.

Theorem 5.5. Suppose that $G$ is amenable. Let $\alpha: G \curvearrowright X$ be an action on a compact metric space. The following are equivalent:

(i) $\alpha$ has the small boundary property,

(ii) there exists an extension $\pi:(Z, \gamma) \rightarrow(X, \alpha)$ which is measure-isomorphic over singleton fibres such that $Z$ is zero-dimensional,

(iii) for every $\varepsilon>0$ there exists a finite collection $\mathscr{O}$ of pairwise disjoint open subsets of $X$ of diameter at most $\varepsilon$ such that $\underline{D}(\bigcup \mathscr{O})=1$,

(iv) for every $\varepsilon>0$ there exists a finite collection $\mathscr{O}$ of pairwise disjoint open subsets of $X$ of diameter at most $\varepsilon$ such that $\underline{D}(\bigcup \mathscr{O}) \geq 1-\varepsilon$.

(v) for every $\varepsilon>0$ and every pair of open sets $U, V \subseteq X$ with $\bar{U} \subseteq V$ there exists an open set $U_{0}$ with $U \subseteq U_{0} \subseteq V$ such that $\bar{D}\left(\partial U_{0}\right) \leq \varepsilon$.

Proof. (i) $\Rightarrow$ (ii). Let us fix a compatible metric $d$ on $X$ for the course of the proof. Given an $n \in \mathbb{N}$ we may find an open cover $\left\{U_{1}, \ldots, U_{m}\right\}$ of $X$ such that for each $j$ the set $U_{j}$ has diameter at most $1 / n$ and $\nu\left(\partial U_{j}\right)=0$ for all $\nu \in M_{G}(X)$. By recursively defining

$$
C_{1}=\bar{U}_{1}, \quad C_{k+1}=\overline{U_{k+1} \backslash\left(\bigcup_{j=1}^{k} C_{k}\right)}
$$

for $k<m$, we obtain a regular closed partition $\mathscr{P}_{n}=\left\{C_{1}, \ldots, C_{m}\right\}$ of $X$. By our choice of the sets $U_{j}$, each $C_{j}$ has diameter at most $1 / n$ and $\nu\left(\partial \mathscr{P}_{n}\right)=0$ for all $\nu \in M_{G}(X)$

Now let $F_{1} \subseteq F_{2} \subseteq \ldots$ be an increasing sequence of finite subsets of $G$ with $e \in F_{n}=F_{n}^{-1}$ and $G=\bigcup_{n=1}^{\infty} F_{n}$. Recursively define a sequence of regular closed partitions $\mathscr{Q}_{n}$ of $X$ by

$$
\mathscr{Q}_{1}=\mathscr{P}_{1}, \quad \mathscr{Q}_{k+1}=\mathscr{P}_{k+1} \vee \bigvee_{s \in F_{k}} \alpha_{s}\left(\mathscr{Q}_{k}\right)
$$

Given $k \in \mathbb{N}$ and $s \in F_{k}$, the partition $\alpha_{s}\left(\mathscr{Q}_{k+1}\right)$ refines $\mathscr{Q}_{k}$ by definition, and we let $r_{k}^{s}: \alpha_{s}\left(\mathscr{Q}_{k+1}\right) \rightarrow \mathscr{Q}_{k}$ be the refinement map sending each member of $\alpha_{s}\left(\mathscr{Q}_{k+1}\right)$ to the member of $\mathscr{Q}_{k}$ which contains it. For brevity, write $r_{k}^{1}=r_{k}$. By the uniqueness of refinement maps between regular closed partitions, we may observe that

$$
r_{k}^{s t} \circ \alpha_{s t} \circ r_{k+1}=r_{k}^{s} \circ \alpha_{s} \circ r_{k+1}^{t} \circ \alpha_{t}
$$

whenever $s, t \in G$ and $k$ are such that all of these maps are defined.

We define a compact metrizable space as the inverse limit

$$
Z=\lim _{\longleftarrow}\left\{\mathscr{Q}_{k}, r_{k}\right\}=\left\{\left(C_{k}\right)_{k \geq 1} \in \prod_{k \geq 1} \mathscr{Q}_{k} \mid C_{k}=r_{k}\left(C_{k+1}\right) \text { for all } k \geq 1\right\}
$$


and denote by $r_{\infty, k}: Z \rightarrow \mathscr{Q}_{k}$ the natural projection map for each $k$. Clearly $Z$ is zerodimensional, and in fact the collection

$$
\mathscr{B}=\left\{r_{\infty, k}^{-1}(A) \mid k \geq 1, A \in \mathscr{Q}_{k}\right\}
$$

is a canonical basis for the topology consisting of clopen sets. Note that each sequence $\left(C_{k}\right)_{k} \in Z$ is uniquely determined by any one of its cofinite tails, which we will use without further mention.

Given an element $z=\left(C_{k}\right)_{k} \in Z$, we observe that the intersection $\bigcap_{k=1}^{\infty} C_{k}$ is nonempty by compactness and can only contain one element because its diameter is at most

$$
\inf _{k \in \mathbb{N}} \operatorname{diam}\left(C_{k}\right) \leq \inf _{k \in \mathbb{N}} 1 / k=0 .
$$

Thus we may define a map $\pi: Z \rightarrow X$ by declaring $\pi\left(\left(C_{k}\right)_{k}\right)$ to be the unique element in the intersection $\bigcap_{k=1}^{\infty} C_{k}$.

It is easy to check that $\pi$ is continuous, for if $z_{1}, z_{2} \in Z$ are both contained in the clopen set $r_{\infty, k}^{-1}(A)$ for some $k \geq 1$ and $A \in \mathscr{Q}_{k}$, then by definition of $\pi$ this means $\pi\left(z_{1}\right), \pi\left(z_{2}\right) \in A$, which by construction of $\mathscr{Q}_{k}$ implies $d\left(\pi\left(z_{1}\right), \pi\left(z_{2}\right)\right) \leq 1 / k$.

The surjectivity of $\pi$ holds because $\left(\mathscr{Q}_{k}\right)_{k}$ is a sequence of successively finer regular closed partitions: if $x \in X$ then there is some $C_{1} \in \mathscr{Q}_{1}$ with $x \in C_{1}$. Inductively, if we have a finite sequence

$$
C_{1} \supseteq C_{2} \supseteq \cdots \supseteq C_{k_{0}} \ni x \quad \text { such that } \quad C_{j} \in \mathscr{Q}_{j}, \quad 1 \leq j \leq k_{0},
$$

then we can find some $C_{k_{0}+1} \in \mathscr{Q}_{k_{0}+1}$ with $x \in C_{k_{0}+1} \subseteq C_{k_{0}}$. This gives us some sequence $\left(C_{k}\right)_{k}=z \in Z$ such that $x=\pi(z)$.

Let us now define a $G$-action $\gamma$ on $Z$. Let $s \in G$. Then there is a $k_{0} \in \mathbb{N}$ such that $s \in F_{k_{0}}$. We define $\gamma_{s}: Z \rightarrow Z$ via the assignment

$$
\left(C_{k}\right)_{k>k_{0}} \mapsto\left(r_{k}^{s}\left(\alpha_{s}\left(C_{k+1}\right)\right)\right)_{k \geq k_{0}} .
$$

Then $\gamma_{s}$ is a well-defined map on $Z$. It is also continuous: if two elements $z_{1}, z_{2} \in Z$ agree on their first $j+1$ components as sequences for $j>k_{0}$, then it follows from the definition of $\gamma_{s}$ that $\gamma_{s}\left(z_{1}\right)$ and $\gamma_{s}\left(z_{2}\right)$ agree on their first $j$ components. As $Z$ is equipped with the product topology coming from $\prod \mathscr{Q}_{k}$, this yields continuity.

It is clear that $\gamma_{e}$ is the identity map. To show that $\gamma$ is an action of $G$ by homeomorphisms, we only need to show that the assignment $s \mapsto \gamma_{s}$ is multiplicative in $G$ with respect to composition. So let $s, t \in G$ and suppose that $s, t, s t \in F_{k_{0}}$ for some $k_{0} \in \mathbb{N}$. Then

$$
\begin{aligned}
\gamma_{s t}\left(\left(C_{k}\right)_{k>k_{0}}\right) & =\left(r_{k}^{s t}\left(\alpha_{s t}\left(C_{k+1}\right)\right)\right)_{k \geq k_{0}} \\
& =\left(r_{k}^{s t}\left(\alpha_{s t}\left(r_{k+1}\left(C_{k+2}\right)\right)\right)\right)_{k \geq k_{0}} \\
& \stackrel{(1)}{=} r_{k}^{s}\left(\alpha_{s}\left(r_{k+1}^{t}\left(\alpha_{t}\left(C_{k+2}\right)\right)\right)\right)_{k \geq k_{0}} \\
& =\gamma_{s}\left(\left(r_{k+1}^{t}\left(\alpha_{t}\left(C_{k+2}\right)\right)\right)_{k \geq k_{0}}\right) \\
& =\left(\gamma_{s} \circ \gamma_{t}\right)\left(\left(C_{k}\right)_{k>k_{0}}\right) .
\end{aligned}
$$

This verifies that $\gamma$ is an action. 
The map $\pi: Z \rightarrow X$ is then $G$-equivariant: for $s \in G, k_{0} \in \mathbb{N}$ as above and arbitrary $z=\left(C_{k}\right)_{k} \in Z$, we have

$$
\alpha_{s}(\pi(z)) \in \alpha_{s}\left(\bigcap_{k=1}^{\infty} C_{k}\right)=\bigcap_{k \geq k_{0}}^{\infty} \alpha_{s}\left(C_{k+1}\right) \subseteq \bigcap_{k \geq k_{0}} r_{k}^{s}\left(\alpha_{s}\left(C_{k+1}\right)\right) \ni \pi\left(\gamma_{s}(z)\right) .
$$

Since the intermediate sets above are one-point sets, this yields $\alpha_{s}(\pi(z))=\pi\left(\gamma_{s}(z)\right)$ for all $z \in Z$ and $s \in G$.

To show that the extension $\pi:(Z, \gamma) \rightarrow(X, \alpha)$ is measure-isomorphic over singleton fibres it suffices to check that $\pi$ satisfies condition (ii) in Proposition 4.3 with respect to the canonical basis $\mathscr{B}$ defined above. Let $W \in \mathscr{B}$ be an element in this basis. Then there are $k \geq 1$ and $A \in \mathscr{Q}_{k}$ such that $W=r_{\infty, k}^{-1}(A)$. The complement is given by $Z \backslash W=$ $\bigsqcup_{C \in \mathscr{Q}_{k}, C \neq A} r_{\infty, k}^{-1}(C)$. By the definition of the map $\pi$, we see that $\pi(W)=A$ and $\pi(Z \backslash W)=$ $\bigcup\left\{C \mid C \in \mathscr{Q}_{k}, C \neq A\right\} \subseteq X$. As $\mathscr{Q}_{k}$ is a regular closed partition the intersection of these two sets lies inside $\partial \mathscr{Q}_{k}$, which has vanishing upper density by construction. Therefore $\bar{D}(\pi(W) \cap \pi(Z \backslash W))=0$ for all $W \in \mathscr{B}$, which finishes the proof of (ii).

(ii) $\Rightarrow$ (iii). Let $\pi:(Z, \gamma) \rightarrow(X, \alpha)$ be an extension as in (ii). Let $\varepsilon>0$ be given. Let $\delta>0$ be small enough so that sets of diameter at most $\delta$ are mapped under $\pi$ to sets of diameter at most $\varepsilon$. Choose a clopen partition $\mathscr{P}$ of $Z$ consisting of sets with diameter at most $\delta$. Let us consider

$$
\mathscr{O}=\{\pi(W) \backslash \pi(Z \backslash W) \mid W \in \mathscr{P}\} .
$$

By design, the members of $\mathscr{O}$ are pairwise disjoint and have diameter at most $\varepsilon$. For every $W \in \mathscr{P}$ we have $\overline{X \backslash \pi(W)} \subseteq \pi(Z \backslash W)$ and hence $\partial \pi(W) \subseteq \pi(W) \cap \pi(Z \backslash W)$, showing that the members of $\mathscr{O}$ are open.

For each $W \in \mathscr{P}$, since $Z \backslash W$ is closed we have $\nu(\pi(W) \cap \pi(Z \backslash W))=0$ for every $\nu \in M_{G}(X)$ by Proposition 4.3(iv). As $X$ is covered by the images of the members of $\mathscr{P}$ under $\pi$, we have

$$
X \backslash \bigcup \mathscr{O} \subseteq \bigcup\{\pi(W) \cap \pi(Z \backslash W) \mid W \in \mathscr{P}\}
$$

This implies that $\underline{D}(\bigcup \mathscr{O})=1-\bar{D}(X \backslash \bigcup \mathscr{O})=1$ and thus yields (iii).

(iii) $\Rightarrow$ (iv). Trivial.

(iv) $\Rightarrow(\mathrm{v})$. Let $U, V \subseteq X$ be open sets with $\bar{U} \subseteq V$. Let $\varepsilon>0$ be given, and assume without loss of generality that it is small enough so that the $\varepsilon$-neighbourhood of $U$ is contained in $V$. By (iv) there is a finite collection $\mathscr{O}$ of pairwise disjoint open subsets of $X$ with diameter less than $\varepsilon$ such that $\underline{D}(\cup \mathscr{O}) \geq 1-\varepsilon$. Set

$$
U_{0}=U \cup \bigcup\{W \in \mathscr{O} \mid W \cap \partial U \neq \emptyset\} .
$$

Then clearly $U \subseteq U_{0} \subseteq V$. By construction we have $\partial U_{0} \subseteq X \backslash \bigcup \mathscr{O}$ and thus $\bar{D}\left(\partial U_{0}\right) \leq \varepsilon$, as desired.

$(\mathrm{v}) \Rightarrow(\mathrm{i})$. Let $U, V \subseteq X$ be open sets such that $\bar{U} \subseteq V$. We will find an open set $W \subseteq X$ with $U \subseteq W \subseteq V$ such that $\bar{D}(\partial W)=0$. Choose a sequence $\left(\varepsilon_{n}\right)_{n}$ of strictly positive real numbers such that $\varepsilon_{n} \rightarrow 0$.

Apply (v) to obtain an open set $W_{1}$ with $U \subseteq W_{1} \subseteq \bar{W}_{1} \subseteq V$ and $\bar{D}\left(\partial W_{1}\right) \leq \varepsilon_{1} / 2$. By Proposition 3.4 there is a closed neighbourhood $Z_{1}$ of $\partial W_{1}$ such that $Z_{1} \subseteq V$ and $\bar{D}\left(Z_{1}\right) \leq \varepsilon_{1}$. Apply (v) again to obtain an open set $W_{2}$ with $W_{1} \subseteq W_{2} \subseteq \bar{W}_{2} \subseteq W_{1} \cup Z_{1}$ 
and $\bar{D}\left(\partial W_{2}\right) \leq \varepsilon_{2} / 2$. By Proposition 3.4 there is a closed neighbourhood $Z_{2}$ of $\partial W_{2}$ such that $Z_{2} \subseteq Z_{1}$ and $\bar{D}\left(Z_{2}\right) \leq \varepsilon_{2}$. Carry on recursively like this to generate for each $n$ an open set $W_{n}$ and a closed neighbourhood $Z_{n}$ of $\partial W_{n}$ such that $U \subseteq W_{n} \subseteq V, W_{n} \subseteq W_{n+1}$, $Z_{n+1} \subseteq Z_{n}, W_{n+1} \subseteq W_{n} \cup Z_{n}$, and $\bar{D}\left(Z_{n}\right) \leq \varepsilon_{n}$. Set

$$
W=\bigcup_{n \in \mathbb{N}} W_{n} .
$$

Then $U \subseteq W \subseteq V$. We also have $\partial W \subseteq \bigcap_{n \in \mathbb{N}} Z_{n}$ and hence $\bar{D}(\partial W)=0$. This proves that $\alpha$ has the small boundary property.

Theorem 5.6. Suppose that $G$ is amenable. Then a free action $\alpha: G \curvearrowright X$ on a compact metrizable space has the small boundary property if and only if it is almost finite in measure.

Proof. The "if" part follows from (iv) $\Rightarrow(i)$ of Theorem 5.5. Let us show the "only if" part. Applying (i) $\Rightarrow$ (ii) of Theorem 5.5, there exists an extension $\pi:(Z, \gamma) \rightarrow(X, \alpha)$ such that $Z$ is zero-dimensional and which is measure-isomorphic over singleton fibres. Since $\alpha$ is free, so is $\gamma$. Fix compatible metrics on $Z$ and $X$.

Let $K$ be a finite subset of $G$ and $\varepsilon>0$. By Theorem 3.13 the action $\gamma$ is almost finite in measure, and so there exists a clopen castle $\left\{\left(V_{i}, S_{i}\right)\right\}_{i \in I}$ with $(K, \varepsilon)$-invariant shapes such that $\underline{D}\left(\bigcup_{i \in I} S_{i} V_{i}\right) \geq 1-\varepsilon$. Choose a $\delta>0$ small enough so that subsets of $Z$ of diameter at most $\delta$ are mapped under $\pi$ to subsets of $X$ of diameter at most $\varepsilon$. By partitioning each $V_{i}$ into smaller clopen sets and relabeling, we may assume that the levels of our castle all have diameter at most $\delta$. For each $i \in I$ set

$$
W_{i}=\pi\left(V_{i}\right) \backslash \pi\left(Z \backslash V_{i}\right)
$$

and note that $s W_{i}=\pi\left(s V_{i}\right) \backslash \pi\left(Z \backslash s V_{i}\right)$ for each $s \in S_{i}$. Then $\left\{\left(W_{i}, S_{i}\right)\right\}_{i \in I}$ is an open castle. The shapes of this castle are $(K, \varepsilon)$-invariant, and its levels have diameter at most $\varepsilon$ by our choice of $\delta$. Also, since $\bar{D}\left(\pi\left(s V_{i}\right) \cap \pi\left(Z \backslash s V_{i}\right)\right)=\bar{D}\left(\pi\left(V_{i}\right) \cap \pi\left(Z \backslash V_{i}\right)\right)=0$ for every $i \in I$ and $s \in S_{i}$ by Propositions 4.3 and 3.3, we have

$$
\underline{D}\left(\bigcup_{i \in I} S_{i} W_{i}\right)=\underline{D}\left(\bigcup_{i \in I} S_{i} V_{i}\right) \geq 1-\varepsilon .
$$

This finishes the proof.

Corollary 5.7. Suppose that $G$ is infinite and amenable. Let $G \curvearrowright X$ be a free action on a compact metrizable space which is almost finite in measure. Then the action has mean dimension zero.

Proof. By Theorem 5.6, the action has the small boundary property. This in turn implies mean dimension zero by Theorem 5.4 of $[33] .^{5}$

\footnotetext{
${ }^{5}$ The arguments there are carried out for $G=\mathbb{Z}$ but the authors make a point that they also apply more generally to all amenable groups.
} 


\section{Comparison And Almost finiteness}

In Theorem 6.1 below we strengthen Theorem 9.2 of [25] using almost finiteness in measure via Theorem 5.6. The novelty is the implication (iii) $\Rightarrow$ (i) under the hypothesis of the small boundary property, which generalizes the corresponding implication in Theorem 9.2 of [25] and is established below in the same way, only this time using Theorem 5.6 instead of the original form of the Ornstein-Weiss tower theorem.

Recall the definitions of almost finiteness and $m$-comparison from Section 2. By Theorem 9.2 of [25], almost finiteness implies comparison, and from this it is readily seen that the action $G \curvearrowright X$ is almost finite if and only if it is almost finite in measure (Definition 3.5) and has comparison.

Theorem 6.1. Suppose that $G$ is amenable. Let $G \curvearrowright X$ be a free action on a compact metrizable space. Then the following are equivalent:

(i) the action is almost finite,

(ii) the action has the small boundary property and comparison,

(iii) the action has the small boundary property and $m$-comparison for some $m \geq 0$.

Proof. The implications (i) $\Rightarrow($ ii $) \Rightarrow$ (iii) are part of Theorem 9.2 of [25], with the small boundary property coming from Theorem 5.6. Let us prove then (iii) $\Rightarrow$ (i).

Note first that if $G$ is finite then the small boundary property implies that $X$ is totally disconnected, in which case the freeness of the action implies that one has an equivariant decomposition $X \cong Y \times G$, where $Y \subseteq X$ is a clopen subset and $G$ acts trivially on the first component and by left translation on the second component. In this case the action is obviously almost finite. We may thus assume that $G$ is infinite.

By hypothesis the action has $m$-comparison for some $m \geq 0$. Let $K$ be a finite subset of $G, \delta>0$, and $n \in \mathbb{N}$. Set $q=(m+1) n$. Since $G$ is infinite there are a finite set $K^{\prime} \subseteq G$ with $K \subseteq K^{\prime}$ and a $\delta^{\prime}>0$ with $\delta^{\prime} \leq \delta$ such that every nonempty $\left(K^{\prime}, \delta^{\prime}\right)$-invariant finite set $F \subseteq G$ is large enough so that there exists a set $S \subseteq F$ satisfying $|F| /(2 q)<|S|<|F| / q$. Since the action has the small boundary property, by Theorem 5.6 it is almost finite in measure, and so we can find an open castle $\left\{\left(V_{i}, T_{i}\right)\right\}_{i \in I}$ with $\left(K^{\prime}, \delta^{\prime}\right)$-invariant shapes such that the remainder $A=X \backslash \bigsqcup_{i \in I} T_{i} V_{i}$ satisfies $\bar{D}(A)<1 /(2 q+1)$. By our choice of $K^{\prime}$ and $\delta^{\prime}$ we can find pairwise disjoint sets $S_{i, 0}, \ldots, S_{i, m} \subseteq T_{i}$ such that each has the same cardinality $\kappa$ satisfying $\left|T_{i}\right| /(2 q)<\kappa<\left|T_{i}\right| / q$. Set $T_{i}^{\prime}=S_{i, 0} \sqcup \cdots \sqcup S_{i, m}$ and note that

$$
\left|T_{i}^{\prime}\right|=(m+1) \kappa<\frac{m+1}{q}\left|T_{i}\right|=\frac{1}{n}\left|T_{i}\right| .
$$

Next set $B=\bigsqcup_{i \in I} S_{i, 0} V_{i}$. By Proposition 3.3, for every $\mu \in M_{G}(X)$ we have $\mu(A) \leq$ $\bar{D}(A)<1 /(2 q+1)$ and hence

$$
\mu(B) \geq \sum_{i \in I} \frac{\left|S_{i, 0}\right|}{\left|T_{i}\right|} \mu\left(T_{i} V_{i}\right)>\frac{1}{2 q} \mu\left(\bigsqcup_{i \in I} T_{i} V_{i}\right)=\frac{1}{2 q}(1-\mu(A))>\mu(A) .
$$

Therefore $A \prec_{m} B$ by our hypothesis of $m$-comparison, and so there exist a finite collection $\mathscr{U}$ of open subsets of $X$ covering $A$, an $s_{U} \in G$ for every $U \in \mathscr{U}$, and a partition $\mathscr{U}=$ $\mathscr{U}_{0} \cup \cdots \cup \mathscr{U}_{m}$ such that for every $i=0, \ldots, m$ the sets $s_{U} U$ for $U \in \mathscr{U}_{i}$ are pairwise disjoint and contained in $B$. 
For every $i \in I$ and $j=0, \ldots, m$ choose a bijection $\varphi_{i, j}: S_{i, 0} \rightarrow S_{i, j}$. For $U \in \mathscr{U}, i \in I$, and $t \in S_{i, 0}$, write $W_{U, i, t}=U \cap s_{U}^{-1} t V_{i}$. Note that each fixed $U$ is partitioned by the open sets $W_{U, i, t}$ for $i \in I$ and $t \in S_{i, 0}$. Also, if for each $U$ we write $j_{U}$ for the $j$ such that $U \in \mathscr{U}_{j}$, then the sets $\varphi_{i, j_{U}}(t) t^{-1} s_{U} W_{U, i, t}$ for $U \in \mathscr{U}, i \in I$, and $t \in S_{i, 0}$ are pairwise disjoint and contained in $\bigsqcup_{i \in I} T_{i}^{\prime} V_{i}$. We have consequently verified that $A \prec \bigsqcup_{i \in I} T_{i}^{\prime} V_{i}$. In conjunction with (2), this establishes almost finiteness.

Recalling the notion of tower dimension for actions from Definition 4.3 of [25], we obtain the following strengthening of Theorem 7.2 in [25]. Note that the assumption of finite covering dimension cannot be removed, as Example 12.5 in [25] illustrates.

Corollary 6.2. Suppose that $G$ is amenable and that $X$ is a compact metrizable space with finite covering dimension. Let $G \curvearrowright X$ be a free minimal action with finite tower dimension. Then the action is almost finite.

Proof. Finite tower dimension and the finite-dimensionality of $X$ together imply, by Theorem 7.2 of [25], that the the action has $m$-comparison for some integer $m \geq 0$. Since the action has the small boundary property in view of the finite-dimensionality of $X$ (as follows for example from Theorem 3.8 of [41]), we can then apply Theorem 6.1 to obtain the conclusion.

\section{Almost Finiteness AND the topological SMAll BOUndary PROPERTY}

In Section 4 we studied extensions which are measure-isomorphic over singleton fibres and used them in Section 5 to establish the equivalence between almost finiteness in measure and the small boundary property. Here we apply such extensions in the more purely topological setting of the so-called topological small boundary property to obtain a result about almost finiteness (Theorem 7.6) that sets the stage for Theorems 8.1 and 8.2 in the next section.

Definition 7.1. Let $G \curvearrowright X$ be an action. A set $A \subseteq X$ is said to be topologically small if there exists a constant $L \in \mathbb{N}$ such that whenever $s_{0}, \ldots, s_{L}$ are distinct elements of $G$ one has $s_{0} A \cap \cdots \cap s_{L} A=\emptyset$. We say that the action has the topological small boundary property if there is a basis for the topology on $X$ consisting of open sets the boundaries of which are topologically small.

Remark 7.2. It is clear from the definition that every topologically small set has vanishing upper density when $G$ is infinite. In particular, the topological small boundary property implies the small boundary property for actions of infinite groups. Note also that the union of finitely many topologically small sets is again topologically small, a fact which will be used without further mention.

By closely examining the proof of (i) $\Rightarrow$ (ii) in Theorem 5.5 one sees that an analogous construction can be used to obtain the following. Since the proof is very similar to before, we omit the details.

Theorem 7.3. Let $\alpha: G \curvearrowright X$ be an action with the topological small boundary property. Then there exists an extension $\pi:(Z, \gamma) \rightarrow(X, \alpha)$ which is measure-isomorphic over singleton fibres such that $Z$ is totally disconnected and for all disjoint clopen sets $W_{1}, W_{2} \subseteq Z$ the intersection $\pi\left(W_{1}\right) \cap \pi\left(W_{2}\right)$ is topologically small. 
Lemma 7.4. Suppose that $G$ is infinite and amenable. Let $G \curvearrowright X$ be a free action with the small boundary property. Let $U \subseteq X$ be an open set with $\underline{D}(U)>0$. Then for every $m \in \mathbb{N}$ and $\eta>0$ there exist pairwise disjoint open sets $U_{1}, \ldots, U_{m} \subseteq U$ satisfying

$$
\underline{D}\left(U_{i}\right) \geq \frac{1}{m} \underline{D}(U)-\eta
$$

for all $i=1, \ldots, m$.

Proof. Let $m \geq 2$ and $\eta>0$ be given with $\eta<\underline{D}(U)$. Setting $\theta=\underline{D}(U)-\eta / 2$, by Proposition 3.4 (taking $A=X \backslash U$ there) we may find an open set $W$ with $\bar{W} \subseteq U$ such that

$$
\underline{D}(W) \geq \theta \text {. }
$$

Let $\delta>0$ be so small that the open $\delta$-neighbourhood of $\bar{W}$ is contained in $U$. As $G$ is infinite, by our choice of $W$ we may find an $\varepsilon>0$ and a finite set $K \subseteq G$ such that every $(K, \varepsilon)$-invariant finite set $S \subseteq G$ has cardinality greater than $4 m(1+\eta) /(\theta \eta)$ and satisfies

$$
\inf _{x \in X} \frac{|S x \cap W|}{|S|} \geq \frac{\theta}{1+\eta},
$$

in which case

$$
\inf _{x \in X}|S x \cap W| \geq \frac{\theta}{1+\eta}|S| \geq \frac{4 m}{\eta} .
$$

Since the action is almost finite in measure by Theorem 5.6, we can find an open castle consisting of towers $\left(V_{j}, S_{j}\right)$ for $j=1, \ldots, N$ such that the shapes $S_{j}$ are $(K, \varepsilon)$-invariant, the levels all have diameter less than $\delta$, and such that

$$
\underline{D}\left(\bigsqcup_{j=1}^{N} S_{j} V_{j}\right) \geq 1-\eta / 4 .
$$

It follows from the diameter condition that every level of the castle is either disjoint from $W$ or lies entirely in $U$. Set

$$
S_{j}^{\prime}=\left\{s \in S_{j}: s V_{j} \cap W \neq \emptyset\right\}
$$

and observe that

$$
\left|S_{j}^{\prime}\right| \geq \inf _{x \in X}\left|S_{j} x \cap W\right| \geq \frac{4 m}{\eta} .
$$

We may then find a partition

$$
S_{j}^{\prime}=S_{j, 1}^{\prime} \sqcup \cdots \sqcup S_{j, m}^{\prime}
$$

with $\left|S_{j, i}^{\prime}\right| \geq\left\lfloor\left|S_{j}^{\prime}\right| / m\right\rfloor$, so that

$$
\frac{\left|S_{j, i}^{\prime}\right|}{\left|S_{j}^{\prime}\right|} \geq \frac{1}{\left|S_{j}^{\prime}\right|}\left(\frac{\left|S_{j}^{\prime}\right|}{m}-1\right) \geq \frac{1}{m}-\frac{\eta}{4}
$$

for each $i=1, \ldots, m$. Set

$$
U_{i}=\bigsqcup_{j=1}^{N} \bigsqcup_{s \in S_{j, i}^{\prime}} s V_{j}
$$


By construction, we have for all $i=1, \ldots, m$ and $\mu \in M_{G}(X)$ that

$$
\begin{aligned}
\mu\left(U_{i}\right)=\sum_{j=1}^{N}\left|S_{j, i}^{\prime}\right| \mu\left(V_{j}\right) & =\sum_{j=1}^{N} \frac{\left|S_{j, i}^{\prime}\right|}{\left|S_{j}^{\prime}\right|}\left|S_{j}^{\prime}\right| \mu\left(V_{j}\right) \\
& \geq\left(\frac{1}{m}-\frac{\eta}{4}\right) \sum_{j=1}^{N}\left|S_{j}^{\prime}\right| \mu\left(V_{j}\right) \\
& \stackrel{(5),(4)}{\geq}\left(\frac{1}{m}-\frac{\eta}{4}\right)\left(\mu(W)-\frac{\eta}{4}\right) .
\end{aligned}
$$

Appealing to Proposition 3.3 we thus conclude that

$$
\begin{aligned}
\underline{D}\left(U_{i}\right) \geq\left(\frac{1}{m}-\frac{\eta}{4}\right)\left(\underline{D}(W)-\frac{\eta}{4}\right) & \stackrel{(3)}{\geq}\left(\frac{1}{m}-\frac{\eta}{4}\right)\left(\underline{D}(U)-\frac{3 \eta}{4}\right) \\
& \geq \frac{1}{m} \underline{D}(U)-\eta .
\end{aligned}
$$

Lemma 7.5. Suppose that $G$ is amenable. Let $G \curvearrowright X$ be a free action with the small boundary property. Let $U \subseteq X$ be an open set with $\underline{D}(U)>0$ and let $C \subseteq X$ be a closed set which is topologically small. Then $C \prec U$.

Proof. Let $L \in \mathbb{N}$ be the smallness constant for $C$ as in Definition 7.1. We will prove the assertion by induction in $L$. First suppose that $L=1$, which means that the images of $C$ under the action of $G$ are pairwise disjoint. As $\underline{D}(U)>0$, there exists some finite set $F \subseteq G$ with $\bigcup_{s \in F} s U=X$. We may assume that $F=F^{-1}$ by replacing $F$ with $F \cup F^{-1}$. Choose an open neighbourhood $V$ of $C$ such that the sets $s V$ for $s \in F$ are pairwise disjoint. Then define $V_{s}=s U \cap V$ for $s \in F$, which yields an open cover of $C$ with the property that the sets $s^{-1} V_{s}$ for $s \in F$ are pairwise disjoint and contained in $U$. This verifies $C \prec U$ for the base step in the induction.

Now assume that the assertion holds for sets with smallness constant at most some given $L \in \mathbb{N}$ and let us show that it holds for sets with smallness constant $L+1$. Let $C \subseteq X$ be a closed topologically small set with smallness constant $L+1$.

Applying Lemma 7.4 repeatedly, we find pairwise disjoint open sets $U_{s} \subset U$ indexed by $s \in G$ with $\underline{D}\left(U_{s}\right)>0$ for all $s$. For every $s \neq e$, the set $C \cap s C$ is clearly topologically small with constant $L$, and so by the induction hypothesis we have $C \cap s C \prec U_{s}$ for all $s \neq e$. For every $s \neq e$ take an open neighbourhood $V_{s}$ of $C \cap s C$ such that $V_{s} \prec U_{s}$, for example the union of a collection of open sets covering $C \cap s C$ which witness the subequivalence $C \cap s C \prec U_{s}$.

We note that the complement $C \backslash \bigcup_{s \in G \backslash\{e\}} s C$ is topologically small with constant $L=1$, and so the same is true of the closed set $C \backslash \bigcup_{s \in G \backslash\{e\}} V_{s}$, which it contains. It follows by the base case of the induction hypothesis that $C \backslash \bigcup_{s \in G \backslash\{e\}} V_{s} \prec U_{e}$. Since

$$
\begin{aligned}
C & =\left(\bigcup_{s \in G \backslash\{e\}} C \cap s C\right) \cup\left(C \backslash\left(\bigcup_{s \in G \backslash\{e\}} s C\right)\right) \\
& \subseteq\left(\bigcup_{s \in G \backslash\{e\}} V_{s}\right) \cup\left(C \backslash \bigcup_{s \in G \backslash\{e\}} V_{s}\right)
\end{aligned}
$$


we conclude using the compactness of $C$ that $C \prec \bigcup_{s \in G} U_{s} \subseteq U$.

Theorem 7.6. Suppose that $G$ is amenable, and that every free action of $G$ on a zerodimensional compact metrizable space is almost finite. Then every free action of $G$ with the topological small boundary property is almost finite.

Proof. Let $X$ be a compact metrizable space and $\alpha: G \curvearrowright X$ a free action with the topological small boundary property. We apply Theorem 7.3 to obtain an extension $\pi:(Z, \gamma) \rightarrow$ $(X, \alpha)$ which is measure-isomorphic over singleton fibres such that $Z$ is zero-dimensional and $\pi\left(W_{1}\right) \cap \pi\left(W_{2}\right)$ is topologically small for all pairs of disjoint clopen sets $W_{1}, W_{2} \subseteq Z$.

As $\alpha$ is free, it is clear that $\gamma$ is free. By assumption, $\gamma$ is almost finite. Since $Z$ is zerodimensional, it follows by the comments in the last paragraph of Section 2 that given an $\varepsilon>0$ and a finite set $K \subseteq G$ we can find a clopen castle in $Z$ consisting of towers $\left(V_{j}, S_{j}\right)$ for $j=1, \ldots, m$ so that each shape $S_{j}$ is $(K, \varepsilon)$-invariant and the castle partitions $Z$. Without loss of generality, we may assume that $e \in S_{j}$ for all $j$. Choose $\delta>0$ small enough so that sets of diameter at most $\delta$ in $Z$ are mapped under $\pi$ to sets of diameter at most $\varepsilon$ in $X$. By subdividing the castle if necessary, we may assume that all of the tower levels have diameter at most $\delta$.

Define

$$
B_{j}=\pi\left(V_{j}\right) \backslash \pi\left(Z \backslash V_{j}\right) .
$$

Then $\left(B_{j}, S_{j}\right)$ is an open tower in $X$ for the action $\alpha$, and together these towers form a castle whose levels have diameter at most $\varepsilon$. The remainder of the castle is contained in the closed set

$$
R=\bigcup_{j=1}^{m} \bigcup_{s \in S_{j}} \pi\left(s V_{j}\right) \cap \pi\left(Z \backslash s V_{j}\right),
$$

which by our choice of $\pi$ is topologically small. Since $\pi$ is measure-isomorphic over singleton fibres, by Proposition 3.3 and the fact that every $G$-invariant Borel probability measure on $Z$ assigns the levels of an open tower the same values we obtain

$$
\underline{D}\left(\bigcup_{j=1}^{m} B_{j}\right)=\underline{D}\left(\bigcup_{j=1}^{m} V_{j}\right) \geq \min _{1 \leq j \leq m} \frac{1}{\left|S_{j}\right|}>0,
$$

so that $R \prec \bigcup_{j=1}^{m} B_{j}$ by Lemma 7.5. Thus $\alpha$ is almost finite.

Corollary 7.7. Suppose that $G$ is amenable, and that every free action of $G$ on a zerodimensional compact metrizable space is almost finite. Then every free action of $G$ on a finite-dimensional compact metrizable space is almost finite.

Proof. This is a consequence of Theorem 7.6 and Theorem 3.8 of [41].

\section{Applications to Classifiability}

Recently in [11] Downarowicz and Zhang showed that if every finitely generated subgroup of $G$ has subexponential growth then every free action of $G$ on a zero-dimensional compact metrizable space is almost finite, i.e., there exist clopen castles which have Følner shapes and partition the space (see the last paragraph of Section 2). Conley, Jackson, Marks, Seward, and Tucker-Drob also observed independently that the tiling argument of [6] can be adapted to the topological setting to establish this fact. In this case one first notes, using 
the clopen version of the Ornstein-Weiss tower theorem (Theorem 3.13), that the problem can be reduced to a matching argument on a bipartite graph. The graph is constructed from a clopen Ornstein-Weiss castle by using a fixed symmetric Følner set to connect points in the leftover part of the space, whose Banach density $d$ is taken to be extremely small, to points in some proportionally small collection of tower levels whose union has Banach density at least $2 d$. With this graph one can progressively build better and better matchings through a process of simultaneously flipping a given matching along a pairwise disjoint collection of augmenting paths. Since subexponential growth implies that there is a uniform upper bound on the length of (minimal) augmenting paths, this process will terminate after finitely many steps, resulting in a clopen matching. One thus obtains a clopen castle which partitions the space, with the apppoximate invariance of the shapes of the original castle being approximately preserved due to the proportional smallness of the collection of tower levels used to build the bipartite graph.

Combined with Corollary 7.7, this result on almost finiteness yields the following theorem. Note that the conclusion is still valid if we replace the hypothesis $\operatorname{dim}(X)<\infty$ with the weaker assumption that the action has the topological small boundary, as we can appeal to Theorem 7.6 instead.

Theorem 8.1. Suppose that every finitely generated subgroup of $G$ has subexponential growth. Let $X$ be a compact metrizable space with finite covering dimension. Then every free action $G \curvearrowright X$ is almost finite.

For a free minimal action $G \curvearrowright X$ on a compact metrizable space, almost finiteness implies that $C(X) \rtimes G$ is $\mathscr{Z}$-stable by Theorem 12.4 of [25] and hence that $C(X) \rtimes G$ has finite nuclear dimension by Theorem $\mathrm{A}$ of [4]. In this case $C(X) \rtimes G$, given that it satisfies the UCT [45], falls under the scope of the classification theorem recorded as Corollary D in [43]. This gives us the following result, which again applies equally well if one assumes the topological small boundary property on the action instead of $\operatorname{dim}(X)<\infty$. The reader may compare this result to Theorem 8.8 of [42].

Theorem 8.2. The crossed products of free minimal actions $G \curvearrowright X$ where $X$ is a compact metrizable space with finite covering dimension and $G$ is such that each of its finitely generated subgroups has subexponential growth are classified by the Elliott invariant (ordered $K$-theory paired with tracial states) and are simple ASH algebras of topological dimension at most 2.

In the case that $G$ is finitely generated and has polynomial growth, one can alternatively establish Theorem 8.1 by substituting the following simpler proof of almost finiteness for free actions on zero-dimensional compact metrizable spaces. We first observe, by considering the word-length metric with respect to a fixed finite generating set and using balls of sufficiently large radius, that such a $G$ satisfies the following property:

(*) there is a constant $c>0$ such that for every finite set $K \subseteq G$ and $\delta>0$ there exists a nonempty finite set $F \subseteq G$ with $\left|F^{-1} F\right| \leq c|F|$ such that both $F$ and $F^{-1} F$ are $(K, \delta)$-invariant.

It then suffices to combine the following two lemmas to obtain the desired conclusion. 
Lemma 8.3. Suppose that $G$ has property (*) with constant $c>0$. Let $G \curvearrowright X$ be a free action on a zero-dimensional compact metrizable space. Then for all clopen sets $A, B \subseteq X$ satisfying $c \bar{D}(A)<\underline{D}(B)$ one has $A \prec B$.

Proof. Let $G \curvearrowright X$ be a free action on a zero-dimensional compact metrizable space and let $A$ and $B$ be clopen subsets of $X$ satisfying $c \bar{D}(A)<\underline{D}(B)$. Choose an $\varepsilon>0$ such that $c(\bar{D}(A)+\varepsilon)<\underline{D}(B)-\varepsilon$.

Take a finite set $K \subseteq G$ and $\delta>0$ such that for every $(K, \delta)$-invariant finite set $E \subseteq G$ one has $\bar{D}(A)+\varepsilon \geq \sup _{x \in X}|A \cap E x| /|E|$ and $\underline{D}(B)-\varepsilon \leq \inf _{x \in X}|B \cap E x| /|E|$. By our choice of $c$, there is a nonempty finite set $F \subseteq G$ such that $\left|F^{-1} F\right| \leq c|F|$ and both $F$ and $F^{-1} F$ are $(K, \delta)$-invariant. Fix an enumeration $s_{1}, \ldots, s_{n}$ of the elements of $F$. Set $A_{1}=A \cap s_{1}^{-1} B$ and for $k=2, \ldots, n$ recursively define

$$
A_{k}=\left(A \backslash\left(A_{1} \cup \cdots \cup A_{k-1}\right)\right) \cap s_{k}^{-1}\left(B \backslash\left(s_{1} A_{1} \cup \cdots \cup s_{k-1} A_{k-1}\right)\right) .
$$

Note that the sets $A_{1}, \ldots, A_{k}$ are clopen, pairwise disjoint, and contained in $A$.

We claim that the union of the sets $A_{1}, \ldots, A_{k}$ is equal to $A$. Suppose to the contrary that there exists an $x \in A \backslash \bigsqcup_{k=1}^{n} A_{k}$. Set $I=\left\{1 \leq i \leq n: s_{i} x \in B\right\}$. Then

$$
|I|=|F x \cap B| \geq(\underline{D}(B)-\varepsilon)|F|
$$

Since $x$ does not belong to any of the sets $A_{1}, \ldots, A_{n}$, for every $i \in I$ there is a $k_{i} \in$ $\{1, \ldots, i-1\}$ and an $x_{i} \in A_{k_{i}}$ such that $s_{i} x=s_{k_{i}} x_{i}$, which we can also write as $x_{i}=s_{k_{i}}^{-1} s_{i} x$. Since the points $s_{1} x, \ldots, s_{n} x$ are distinct by freeness and the sets $A_{1}, \ldots, A_{n}$ are pairwise disjoint, the points $x_{i}$ for $i \in I$ are distinct, which implies that the group elements $s_{k_{i}}^{-1} s_{i}$ for $i \in I$ are distinct. Thus

$$
\begin{aligned}
|I| \leq\left|A \cap F^{-1} F x\right| & \leq(\bar{D}(A)+\varepsilon)\left|F^{-1} F\right| \\
& \leq(\bar{D}(A)+\varepsilon) c|F| \\
& <(\underline{D}(B)-\varepsilon)|F|,
\end{aligned}
$$

contradicting (6). We must therefore have $A=\bigsqcup_{k=1}^{n} A_{k}$. Since the sets $s_{1} A_{1}, \ldots, s_{n} A_{n}$ are pairwise disjoint and contained in $B$, we have thus shown that $A \prec B$.

Lemma 8.4. Suppose that $G$ is amenable. Let $G \curvearrowright X$ be a free action on a zero-dimensional compact metrizable space. Suppose that there exists a $c>0$ such that for all clopen sets $A, B \subseteq X$ satisfying $c \bar{D}(A) \leq \underline{D}(B)$ one has $A \prec B$. Then the action is almost finite.

Proof. It is clear that the hypothesis in the theorem statement can be substituted for $m$ comparison in the proof of (iii) $\Rightarrow$ (i) in Theorem 6.1 given that the castle there can be taken to be clopen, as the proof of Theorem 3.13 demonstrates.

Remark 8.5. Finally we point out that, for finitely generated groups, property $(*)$ is actually equivalent to polynomial growth. Indeed for finitely generated groups polynomial growth is equivalent to virtual nilpotence by Gromov's theorem [17], and property $(*)$ (or even a weaker version of it, known as the Tempelman condition, which does not require $F^{-1} F$ to be $(K, \delta)$-invariant) implies virtual nilpotence by Corollary 11.2 in [3]. This corollary shows that if $G$ is a finitely generated group for which there is a $b>0$ such that for every finite set $E \subseteq G$ there exists a finite set $F \subseteq G$ satisfying $\left|F^{-1} F\right| \leq b|F|$ and $E \subseteq F$, then $G$ is virtually nilpotent (the corollary is stated in [3] with $F^{2}$ in place of $F^{-1} F$, but the authors' 
application of their Corollary 1.7 in the proof works equally well in the latter case). While the containment condition $E \subseteq F$ for a given $E$ does not appear in the definition of property $(*)$, we can arrange for the set $F$ in the definition to satisfy this condition, for if $F$ is $(E, \delta)$ invariant for a sufficiently small $\delta>0$ then it contains $E s$ for some $s \in G$, as is easy to check, in which case $F s^{-1}$ contains $E$ and satisfies $\left|\left(F s^{-1}\right)^{-1} F s^{-1}\right|=\left|F^{-1} F\right|$ and $\left|F s^{-1}\right|=|F|$.

\section{Crossed products And the Toms-Winter Conjecture}

The Toms-Winter conjecture asserts the equivalence of the following three conditions for simple separable infinite-dimensional unital nuclear $\mathrm{C}^{*}$-algebras:

(i) finite nuclear dimension,

(ii) $\mathscr{Z}$-stability,

(iii) strict comparison.

Recently in [5] the conjecture was settled in the case that the $\mathrm{C}^{*}$-algebra has uniform property $\Gamma$, as defined below. Since $\mathscr{Z}$-stability implies uniform property $\Gamma$ this yields the implication (ii) $\Rightarrow$ (i) in full generality. As finite nuclear dimension was known to imply $\mathscr{Z}$-stability [46] one thereby obtains the equivalence of (i) and (ii).

Definition 9.1. A unital $\mathrm{C}^{*}$-algebra $A$ with nonempty tracial state space is said to have uniform property $\Gamma$ if for every finite set $\Omega \subseteq A$ and $\varepsilon>0$ there are two orthogonal positive contractions $e_{1}, e_{2} \in A$ such that for all $a \in \Omega$ and $k=1,2$ one has

(i) $\left\|e_{k} a-a e_{k}\right\|<\varepsilon$, and

(ii) $\left|\tau\left(e_{k} a\right)-\frac{1}{2} \tau(a)\right|<\varepsilon$ for every tracial state $\tau$ on $A$.

In condition (i) above one can equivalently use the uniform trace norm $\|\cdot\|_{2, \mathrm{u}}$ (the supremum of the trace norms over all tracial states), as follows for example from Propositions 4.5 and 4.6 in [28].

Making use of tiling techniques as in the proofs of Theorem 5.3 in [6] and Theorem 12.4 in [25] we will verify uniform property $\Gamma$ for crossed products of free actions with the small boundary property, i.e., which are almost finite in measure. The basic tool is the following disjointified version of the Ornstein-Weiss quasitiling theorem. We say that a set $E \subseteq G$ is tiled by a collection $\mathscr{T}$ of subsets of $G$ if $E$ can be partitioned into sets of the form $T c$ where $T \in \mathscr{T}$ and $c \in G$.

Lemma 9.2. Suppose that $G$ is amenable. Let $K$ be a finite subset of $G$ and $\delta>0$. Let $0<\varepsilon<\frac{1}{2}$. Then there are a finite set $K^{\prime} \subseteq G, a \delta^{\prime}>0$, and a finite collection $\mathscr{T}$ of $(K, \delta)$-invariant finite subsets of $G$ such that for every $\left(K^{\prime}, \delta^{\prime}\right)$-invariant finite set $E \subseteq G$ there is a set $E^{\prime} \subseteq E$ such that $\left|E^{\prime}\right| \geq(1-\varepsilon)|E|$ and $E^{\prime}$ can be tiled by $\mathscr{T}$.

Proof. As is simple to check, there exists an $\eta>0$ such that for every $(K, \eta)$-invariant finite set $T \subseteq G$ and every $T^{\prime} \subseteq T$ with $\left|T^{\prime}\right| \geq(1-\eta)|T|$ the set $T^{\prime}$ is $(K, \delta)$-invariant. By shrinking $\varepsilon$ if necessary we may assume that $\varepsilon \leq \eta$. By the Ornstein-Weiss quasitiling theorem (see Theorem 4.36 of [26]), there are a finite set $K^{\prime} \subseteq G$, a $\delta^{\prime}>0$, and $(K, \eta)$ invariant finite sets $T_{1}, \ldots, T_{m} \subseteq G$ such that for every $\left(K^{\prime}, \delta^{\prime}\right)$-invariant finite set $E \subseteq G$ there exist $C_{1}, \ldots, C_{m} \subseteq G$ and pairwise disjoint sets $T_{i, c} \subseteq T_{i}$ with $\left|T_{i, c}\right| \geq(1-\eta)\left|T_{i}\right|$ for $i=1, \ldots, m$ and $c \in C_{i}$ such that

(i) $\bigcup_{i=1}^{m} T_{i} C_{i}$ is a subset of $E$ with cardinality at least $(1-\varepsilon)|E|$, and 
(ii) $\bigsqcup_{i=1}^{m} \bigsqcup_{c \in C_{i}} T_{i, c} c=\bigcup_{i=1}^{m} T_{i} C_{i}$.

By our choice of $\eta$, each of the sets $T_{i, c}$ is $(K, \delta)$-invariant. We can thus take $\mathscr{T}$ to be the union of all sets $T \subseteq G$ for which there is an $1 \leq i \leq n$ such that $T \subseteq T_{i}$ and $|T| \geq(1-\eta)\left|T_{i}\right|$.

Lemma 9.3. Let $X$ be a compact metrizable space and $G \curvearrowright X$ a free action which is almost finite in measure. Let $\mathscr{P}$ be a finite regular closed partition of $X$ whose boundary $\partial \mathscr{P}$ (Definition 5.2) has zero upper density. Let $K$ be a finite subset of $G$ and $\delta>0$. Then there is an open castle $\left\{\left(V_{i}, S_{i}\right)\right\}_{i \in I}$ such that

(i) each shape $S_{i}$ is $(K, \delta)$-invariant,

(ii) each level of the castle is contained in the interior of some member of $\mathscr{P}$ and has boundary of upper density zero,

(iii) $\underline{D}\left(\bigsqcup_{i \in I} S_{i} V_{i}\right) \geq 1-\varepsilon$.

Proof. By almost finiteness in measure there is an open castle $\left\{\left(V_{i}, S_{i}\right)\right\}_{i \in I}$ such that each shape $S_{i}$ is $(K, \delta)$-invariant and $\underline{D}\left(\bigsqcup_{i \in I} S_{i} V_{i}\right) \geq 1-\varepsilon$. The proof of Theorem 5.6 shows that we may assume that the boundary of each level of the castle has upper density zero, which is equivalent to saying that $\bar{D}\left(\partial \bigsqcup_{i \in I} S_{i} V_{i}\right)=0$. Write the interiors of the members of $\mathscr{P}$ as $A_{1}, \ldots, A_{n}$. Set $A_{0}=X \backslash\left(A_{1} \sqcup \cdots \sqcup A_{n}\right)$. Then the sets $A_{0}, \ldots, A_{n}$ partition $X$. Let $i \in I$. For each $x \in V_{i}$ consider the function $\sigma_{x} \in\{0, \ldots, n\}^{S_{i}}$ such that $s x \in A_{\sigma_{x}(s)}$ for every $s \in S_{i}$. Write $\Sigma_{i}=\{1, \ldots, n\}^{S_{i}}$. For every $\sigma \in \Sigma_{i}$ define the set $V_{i, \sigma}=\left\{x \in V_{i}: \sigma_{x}=\sigma\right\}$, which is open because the sets $A_{1}, \ldots, A_{n}$ are open. The pairs $\left(V_{i, \sigma}, S_{i}\right)$ for $\sigma \in \Sigma_{i}$ thus form an open castle each of whose levels belongs to the interior of some member of $\mathscr{P}$, and the complement $S_{i} V_{i} \backslash \bigsqcup_{\sigma \in \Sigma_{i}} S_{i} V_{i, \sigma}$ has upper density zero given that it is contained in $S_{i} S_{i}^{-1} \partial \mathscr{P}$. Having done this for each $i \in I$, we obtain an open castle $\left\{\left(V_{i, \sigma}, S_{i}\right): i \in\right.$ $\left.I, \sigma \in \Sigma_{i}\right\}$ such that the complement of the union of its levels is contained in the union of $X \backslash \bigsqcup_{i \in I} S_{i} V_{i}$ and $\bigcup_{i \in I} S_{i} S_{i}^{-1} \partial \mathscr{P}$, and since the latter has upper density zero we deduce that $\underline{D}\left(\bigsqcup_{i \in I} \bigsqcup_{\sigma \in \Sigma_{i}} S_{i} V_{i, \sigma}\right)=\underline{D}\left(\bigsqcup_{i \in I} S_{i} V_{i}\right) \geq 1-\varepsilon$. Finally, since the boundary of the set $\bigsqcup_{i \in I} \bigsqcup_{\sigma \in \Sigma_{i}} S_{i} V_{i, \sigma}$ is contained in the union of $\partial \bigsqcup_{i \in I} S_{i} V_{i}$ and $\bigcup_{i \in I} S_{i} S_{i}^{-1} \partial \mathscr{P}$, it must have upper density zero, so that the boundary of each level of the castle we have constructed has upper density zero. Our requirements are thus fulfilled.

In the proof of the following theorem we will abuse notation and use the same symbol $\mu$ to denote a measure and the integral of a function with respect to that measure, as well as the induced tracial state on the crossed product.

Theorem 9.4. Suppose that $G$ is infinite and amenable. Let $X$ be a compact metrizable space and $G \curvearrowright X$ a free action with the small boundary property. Then $C(X) \rtimes G$ has uniform property $\Gamma$.

Proof. To prove the theorem we will show that for all finite sets $\Omega \subseteq C(X) \rtimes G$ and $\varepsilon>0$ there exist positive contractions $f_{1}, f_{2} \in C(X)$ with disjoint supports such that

$$
\left|\mu\left(f_{k} a\right)-\frac{1}{2} \mu(a)\right|<\varepsilon
$$

for all $k=1,2, a \in \Omega$, and $\mu \in M_{G}(X)$, and

$$
\left\|f_{k} a-a f_{k}\right\|<\varepsilon
$$

for all $k=1,2$ and $a \in \Omega$. 
First of all, we claim that it is enough to show that for every finite regular closed partition $\mathscr{P}$ of $X$ whose boundary has upper density zero (Definition 5.2), every finite set $L \subseteq G$, and every $\varepsilon>0$ we can find positive contractions $f_{1}, f_{2} \in C(X)$ with disjoint supports such that

$$
\left|\mu\left(f_{k} 1_{A}\right)-\frac{1}{2} \mu(A)\right|<\varepsilon
$$

for all $k=1,2, A \in \mathscr{P}$, and $\mu \in M_{G}(X)$, and, denoting by $u_{s}$ the canonical unitary in $C(X) \rtimes G$ associated to a given group element $s$,

$$
\left\|f_{k} u_{s}-u_{s} f_{k}\right\|<\varepsilon
$$

for all $k=1,2$ and $s \in L$.

Indeed first note that to verify (7) it is enough that $\Omega$ be a subset of $C(X)$, for if $E$ denotes the canonical conditional expectation from $C(X) \rtimes G$ onto $C(X)$ then given any $f \in C(X)$ and $a \in C(X) \rtimes G$ we have $\mu(f a)=\mu(E(f a))=\mu(f E(a))$. Note next that by Theorem 5.5 there is an extension $G \curvearrowright Z$ of $G \curvearrowright X$ which is measure-isomorphic over singleton fibres such that $Z$ is zero-dimensional. Accordingly we can view $C(X)$ as a $G$-invariant unital $\mathrm{C}^{*}$-subalgebra of $C(Z)$ and $C(X) \rtimes G$ as a unital $\mathrm{C}^{*}$-subalgebra of $C(Z) \rtimes G$. By zerodimensionality, for every finite collection of functions in $C(Z)$, and in particular for every finite collection of functions in $C(X)$, there is a clopen partition $\mathscr{P}$ of $Z$ such that each function in the collection is approximately equal in norm, to within a prescribed tolerance, to a linear combination of the indicator functions of the members of $\mathscr{P}$. Given that the factor map $Z \rightarrow X$ induces a bijection $M_{G}(Z) \rightarrow M_{G}(X)$ via its push-forward, a simple approximation argument then shows that to verify (7) we may in fact go outside of $C(X)$ within the larger algebra $C(Z)$ and instead quantify $\Omega$ over the collections $\left\{1_{A}: A \in \mathscr{P}\right\}$ where $\mathscr{P}$ ranges over the clopen partitions of $Z$. But this then means that it is enough to verify (9) with $\mathscr{P}$ ranging, as in the claim, over the finite regular closed partitions of $X$ whose boundaries have upper density zero, for under the factor map $Z \rightarrow X$ the image of every clopen partition of $Z$ is a regular closed partition of $X$ whose boundary has upper density zero, as the proof of Proposition $5.5(\mathrm{ii}) \Rightarrow(\mathrm{iii})$ shows. Finally, given that $C(X)$ is a commutative $\mathrm{C}^{*}$-algebra one can replace (8) by (10) via a simple approximation argument, thereby establishing the claim.

So now let $\mathscr{P}$ be a regular closed partition of $X$ with $\bar{D}(\partial \mathscr{P})=0, L$ a finite symmetric subset of $G$, and $0<\varepsilon<1$. Choose an integer $Q>1 / \varepsilon$. By amenability there are a finite set $K \subseteq G$ and a $\delta>0$ such that every $(K, \delta)$-invariant finite set $E \subseteq G$ satisfies $\left|\bigcap_{s \in L^{Q}} s E\right| \geq(1-\varepsilon / 4)|E|$. By Lemma 9.2 there are a finite set $K^{\prime} \subseteq G$, a $\delta^{\prime}>0$, and a finite collection $\mathscr{T}=\left\{T_{1}, \ldots, T_{J}\right\}$ of $(K, \delta)$-invariant finite subsets of $G$ such that for every $\left(K^{\prime}, \delta^{\prime}\right)$-invariant finite set $E \subseteq G$ there is a set $E^{\prime} \subseteq E$ such that $\left|E^{\prime}\right| \geq(1-\varepsilon / 6)|E|$ and $E^{\prime}$ can be tiled by $\mathscr{T}$. Set $M=\max _{1 \leq j \leq J}\left|T_{j}\right|$.

By Theorem 5.6, the action $G \curvearrowright X$ is almost finite in measure, so by Lemma 9.3 there is an open castle $\left\{\left(V_{i}, S_{i}\right)\right\}_{i \in I}$ such that

(i) each shape $S_{i}$ is $\left(K^{\prime}, \delta^{\prime}\right)$-invariant,

(ii) each level of the castle is contained in the interior of some member of $\mathscr{P}$ and has boundary of upper density zero, and

(iii) $\underline{D}\left(\bigsqcup_{i \in I} S_{i} V_{i}\right) \geq 1-\varepsilon / 6$.

As $G$ is infinite, we may demand even stronger approximate invariance in (i) so as to additionally force each of the shapes $S_{i}$ to have cardinality at least $6 J M|\mathscr{P}|^{M} / \varepsilon$. 
Let $i \in I$. By our invocation of Lemma 9.2 , there is a set $S_{i}^{\prime} \subseteq S_{i}$ with $\left|S_{i}^{\prime}\right| \geq(1-\varepsilon / 6)\left|S_{i}\right|$ and sets $C_{i, 1}, \ldots, C_{i, J} \subseteq G$ such that the collection $\left\{T_{j} c: 1 \leq j \leq J, c \in C_{i, j}\right\}$ partitions $S_{i}^{\prime}$. Let $1 \leq j \leq J$. For each $c \in C_{i, j}$ write $\sigma_{c}$ for the element of $\mathscr{P}^{T_{j}}$ such that $t c V_{i} \subseteq \sigma_{c}(t)$ for all $t \in T_{j}$. This element is unique by (ii) and the fact that the interiors of the members of $\mathscr{P}$ are pairwise disjoint by the definition of regular closed partition. For each $\sigma \in \mathscr{P} T_{j}$ write $C_{i, j, \sigma}$ for the set of all $c \in C_{i, j}$ such that $\sigma_{c}=\sigma$, and choose two disjoint subsets $C_{i, j, \sigma}^{(1)}$ and $C_{i, j, \sigma}^{(2)}$ of $C_{i, j, \sigma}$ with equal cardinality such that the complement of their union in $C_{i, j, \sigma}$, which we denote by $C_{i, j, \sigma}^{(0)}$, is either empty or a singleton, depending on the parity of the cardinality of $C_{i, j, \sigma}$.

Set $T_{j, Q}=\bigcap_{s \in L^{Q}} s T_{j}$, and note that, by our choice of $K$ and $\delta$,

$$
\left|T_{j, Q}\right| \geq(1-\varepsilon / 4)\left|T_{j}\right|
$$

Next recursively define, for $q=0, \ldots, Q-1$,

$$
T_{j, q}=L^{Q-q} T_{j, Q} \backslash L^{Q-q-1} T_{j, Q}
$$

The sets $T_{j, q}$ for $q=0, \ldots, Q$ are pairwise disjoint and contained in $T_{j}$, and for $s \in L$ we have

$$
s T_{j, Q} \subseteq T_{j, Q-1} \cup T_{j, Q}
$$

and, for $q=1, \ldots, Q$,

$$
s T_{j, q} \subseteq T_{j, q-1} \cup T_{j, q} \cup T_{j, q+1}
$$

Let $i \in I, 1 \leq j \leq J$, and $c \in C_{i, j}$ be given. Since the boundaries of our towers have upper density zero, by Proposition 3.4 there is an $\eta>0$ such that the set $B_{i, j, c}:=\left\{x \in c V_{i}\right.$ : $\left.d\left(x, X \backslash c V_{i}\right)<\eta\right\}$ satisfies

$$
\mu\left(B_{i, j, c}\right)<\frac{\varepsilon}{4 \sum_{i \in I} \sum_{j=1}^{J}\left|C_{i, j}\right|}
$$

for all $\mu \in M_{G}(X)$. Set $W_{i, j, c}=c V_{i} \backslash B_{i, j, c}$, which is closed. By Urysohn's lemma there is a continuous function $g_{i, j, c}: X \rightarrow[0,1]$ which is zero on the complement of $c V_{i}$ and one on $W_{i, j, c}$. Given $1 \leq j \leq J$ and $c \in C_{i, j}$ and writing $\alpha_{t}$ for the automorphism of $C(X)$ that composes functions with the transformation $x \mapsto t^{-1} x$, we then define the function

$$
\tilde{g}_{i, j, c}=\sum_{q=0}^{Q} \sum_{t \in T_{j, q}} \frac{q}{Q} \alpha_{t}\left(g_{i, j, c}\right) .
$$

Now set

$$
f_{1}=\sum_{i \in I} \sum_{j=1}^{J} \sum_{c \in C_{i, j, \sigma}^{(1)}} \tilde{g}_{i, j, c}, \quad f_{2}=\sum_{i \in I} \sum_{j=1}^{J} \sum_{c \in C_{i, j, \sigma}^{(2)}} \tilde{g}_{i, j, c} .
$$

For every $s \in L$ we have

$$
u_{s} \tilde{g}_{i, j, c} u_{s}^{-1}-\tilde{g}_{i, j, c}=\sum_{q=0}^{Q} \sum_{t \in T_{j, q}} \frac{q}{Q} \alpha_{s t}\left(g_{i, j, c}\right)-\sum_{q=0}^{Q} \sum_{t \in T_{j, q}} \frac{q}{Q} \alpha_{t}\left(g_{i, j, c}\right)
$$


and so the function $u_{s} \tilde{g}_{i, j, c} u_{s}^{-1}-\tilde{g}_{i, j, c}$ has norm at most $1 / Q$ by (12) and (13). Since these functions for different $i, j$ and $c$ have pairwise disjoint supports, we deduce that

$$
\left\|u_{s} f_{k} u_{s}^{-1}-f_{k}\right\| \leq \frac{1}{Q}<\varepsilon
$$

for $k=1,2$, verifying the requirement (10).

Finally, we check (9). For $k=1,2$ we set

$$
Z_{k}=\bigsqcup_{i \in I}^{J} \bigsqcup_{j=1} \bigsqcup_{\sigma \in \mathscr{P}^{T_{j}}} T_{c \in C_{i, j, \sigma}^{(k)}} c V_{i}, \quad \tilde{Z}_{k}=\bigsqcup_{i \in I}^{J} \bigsqcup_{j=1}^{J} \bigsqcup_{\sigma \in \mathscr{P}^{T_{j}}} \bigsqcup_{c \in C_{i, j, \sigma}^{(k)}} T_{j} c W_{i, j, c} .
$$

Let $A \in \mathscr{P}, \mu \in M_{G}(X)$, and $k \in\{1,2\}$. Note first that $\mu\left(A \cap Z_{1}\right)=\mu\left(A \cap Z_{2}\right)$ as we chose the sets $C_{i, j, \sigma}^{(1)}$ and $C_{i, j, \sigma}^{(2)}$ to always have equal cardinality. Thus

$$
\mu\left(f_{k} 1_{A}\right) \leq \mu\left(A \cap Z_{k}\right)=\frac{1}{2} \mu\left(A \cap\left(Z_{1} \sqcup Z_{2}\right)\right) \leq \frac{1}{2} \mu(A) .
$$

Next set $R_{0}=\bigsqcup_{i \in I} \bigsqcup_{j=1}^{J} \bigsqcup_{\sigma \in \mathscr{P}^{T}} \bigsqcup_{c \in C_{i, j, \sigma}^{(0)}} T_{j} c V_{i}$ and observe that for every $i \in I$ we have

$$
\left|\bigsqcup_{j=1}^{J} \bigsqcup_{\sigma \in \mathscr{P}^{T} j} \bigsqcup_{c \in C_{i, j, \sigma}^{(0)}} T_{j} c\right| \leq J M|\mathscr{P}|^{M} \leq \frac{\varepsilon}{6}\left|S_{i}\right|
$$

and hence $\mu\left(R_{0}\right) \leq \varepsilon / 6$. Therefore

$$
\begin{aligned}
\mu\left(X \backslash\left(Z_{1} \sqcup Z_{2}\right)\right) & \leq \mu\left(R_{0}\right)+\mu\left(\bigsqcup_{i \in I}\left(S_{i} \backslash S_{i}^{\prime}\right) V_{i}\right)+\mu\left(X \backslash \bigsqcup_{i \in I} S_{i} V_{i}\right) \\
& \leq \frac{\varepsilon}{6}+\frac{\varepsilon}{6}+\frac{\varepsilon}{6} \leq \frac{\varepsilon}{2}
\end{aligned}
$$

and so

$$
\mu\left(A \cap Z_{k}\right)=\frac{1}{2} \mu\left(A \cap\left(Z_{1} \sqcup Z_{2}\right)\right) \geq \frac{1}{2} \mu(A)-\frac{\varepsilon}{2} .
$$

Since the set $R_{k}=\bigsqcup_{i \in I} \bigsqcup_{j=1}^{J} \bigsqcup_{\sigma \in \mathscr{P}^{T}} \bigsqcup_{c \in C_{i, j, \sigma}^{(k)}}\left(T_{j} \backslash T_{j, Q}\right) c V_{i}$ satisfies $\mu\left(R_{k}\right) \leq \varepsilon / 4$ by (11), and we have $\mu\left(Z_{k} \backslash \tilde{Z}_{k}\right)<\varepsilon / 4$ by (14), it follows that

$$
\begin{aligned}
\mu\left(f_{k} 1_{A}\right) \geq \mu\left(\left(A \cap \tilde{Z}_{k}\right) \backslash R_{k}\right) & \geq \mu\left(A \cap Z_{k}\right)-\mu\left(Z_{k} \backslash \tilde{Z}_{k}\right)-\mu\left(R_{k}\right) \\
& \geq \frac{1}{2} \mu(A)-\varepsilon .
\end{aligned}
$$

Combined with (15) this yields $\left|\mu\left(f_{k} 1_{A}\right)-\frac{1}{2} \mu(A)\right|<\varepsilon$, verifying (9). This finishes the proof.

In view of [5] we deduce the following corollary.

Corollary 9.5. The Toms-Winter conjecture holds for the simple crossed product $C^{*}$-algebras in the statement of Theorem 9.4. 
We expect that the conditions in the Toms-Winter conjecture are actually satisfied by all of the simple crossed products in the statement of Theorem 9.4. This is known to be the case when $G=\mathbb{Z}$ by the work of Elliott and Niu [13].

Remark 9.6. It is worth pointing out that the proof of Theorem 9.4 verifies a version of uniform property $\Gamma$ for the Cartan pair given by the inclusion $C(X) \subseteq C(X) \rtimes G$. In other words, the elements witnessing uniform property $\Gamma$ for the crossed products in Theorem 9.4 are actually coming from the diagonal subalgebra $C(X)$.

Stuart White has pointed out to us that the analogous property for Cartan pairs in von Neumann algebras has been studied before, and in fact played a crucial role in the first example of a $\mathrm{II}_{1}$-factor with two nonconjugate Cartan subalgebras, due to Connes and Jones [8]. In particular, it is known in the von Neumann algebra context that property $\Gamma$ for a Cartan pair can fail even if the ambient algebra itself has property $\Gamma$.

This gives rise to the speculation that uniform property $\Gamma$ for the $\mathrm{C}^{*}$-algebraic Cartan pair $C(X) \subseteq C(X) \rtimes G$ may be closely tied to the small boundary property of the action, whereas we would expect that uniform property $\Gamma$ for the crossed product $\mathrm{C}^{*}$-algebra alone may hold without any assumptions on the action used to construct it besides freeness.

\section{REFERENCES}

[1] A. Bartels. Coarse flow spaces for relatively hyperbolic groups. Compositio Math. 153 (2017), 745-779.

[2] J. Bosa, N. Brown, Y. Sato, A. Tikuisis, S. White and W. Winter. Covering dimension of $\mathrm{C}^{*}$-algebras and 2-coloured classification. Mem. Amer. Math. Soc. 257 (2019), 1-97.

[3] E. Breuillard, B. Green, and T. Tao. The structure of approximate groups. Publ. Math. Inst. Hautes Études Sci. 116 (2012), 115-221.

[4] J. Castillejos, S. Evington, A. Tikuisis, S. White, and W. Winter. Nuclear dimension of simple $\mathrm{C}^{*}-$ algebras. arxiv:1901.05853.

[5] J. Castillejos et al. Strict closures of nuclear $\mathrm{C}^{*}$-algebras and uniform property $\Gamma$. In preparation.

[6] C. Conley, S. Jackson, D. Kerr, A. Marks, B. Seward, and R. Tucker-Drob. Følner tilings for actions of amenable groups. Math. Ann. 371 (2018), 663-683.

[7] A. Connes. Classification of injective factors. Cases $\mathrm{II}_{1}, \mathrm{II}_{\infty}, \mathrm{III}_{\lambda}, \lambda \neq 1$. Ann. of Math. (2) 104 (1976), $73-115$.

[8] A. Connes and V. F. R. Jones: A II 1 factor with two nonconjugate Cartan subalgebras. Bull. Amer. Math. Soc. 6 (1982), 211-212.

[9] T. Downarowicz and E. Glasner. Isomorphic extensions and applications. Topol. Methods Nonlinear Anal. 48 (2016), 321-338.

[10] T. Downarowicz, D. Huczek, and G. Zhang. Tilings of amenable groups. J. reine angew. Math. 747 (2019), 277-298.

[11] T. Downarowicz and G. Zhang. The comparison property of amenable groups. arXiv:1712.05129.

[12] G. Elliott, G. Gong, H. Lin, and Z. Niu. On the classification of simple amenable $\mathrm{C}^{*}$-algebras with finite decomposition rank, II. arXiv:1507.03437.

[13] G. A. Elliott and Z. Niu. The $\mathrm{C}^{*}$-algebra of a minimal homeomorphism of zero mean dimension. Duke Math. J. 166 (2017), 3569-3594.

[14] J. Giol and D. Kerr. Subshifts and perforation. J. reine angew. Math. 639 (2010), 107-119.

[15] G. Gong, H. Lin, and Z. Niu. Classification of finite simple amenable $\mathscr{Z}$-stable $\mathrm{C}^{*}$-algebras. arXiv:1501.00135.

[16] R. Grigorchuk and I. Pak. Groups of intermediate growth: an introduction. Enseign. Math. (2) 54 (2008), 251-272.

[17] M. Gromov. Groups of polynomial growth and expanding maps. Inst. Hautes Études Sci. Publ. Math. 53 (1981), 53-73. 
[18] M. Gromov. Topological invariants of dynamical systems and spaces of holomorphic maps: I. Math. Phys. Anal. Geom. 2 (1999) 323-415.

[19] E. Guentner, R. Willett, and G. Yu. Dynamic asymptotic dimension: relation to dynamics, topology, coarse geometry, and $\mathrm{C}^{*}$-algebras. Math. Ann. 367 (2017), 785-829.

[20] Y. Gutman. Embedding $\mathbb{Z}^{k}$-actions in cubical shifts and $\mathbb{Z}^{k}$-symbolic extensions. Ergodic Theory Dynam. Systems 31 (2011), 383-403.

[21] Y. Gutman. Embedding topological dynamical systems with periodic points in cubical shifts. Ergodic Theory Dynam. Systems 37 (2017), 512-538.

[22] Y. Gutman, E. Lindenstrauss and M. Tsukamoto. Mean dimension of $\mathbb{Z}^{k}$-actions. Geom. Funct. Anal. 26 (2016), 778-817.

[23] I. Hirshberg and J. Orovitz. Tracially $\mathscr{Z}$-absorbing $\mathrm{C}^{*}$-algebras. J. Funct. Anal. 265 (2013), 765-785.

[24] A. S. Kechris. Classical Descriptive Set Theory. Graduate Texts in Mathematics, 156. Springer-Verlag, New York, 1995.

[25] D. Kerr. Dimension, comparison, and almost finiteness. To appear in J. Eur. Math. Soc. arxiv:1710.00393.

[26] D. Kerr and H. Li. Ergodic Theory: Independence and Dichotomies. Springer, Cham, 2016.

[27] E. Kirchberg. The classification of purely infinite C*-algebras using Kasparov's theory. Preprint, 1994.

[28] E. Kirchberg and M. Rørdam. Central sequence $\mathrm{C}^{*}$-algebras and tensorial absorption of the Jiang-Su algebra. J. reine angew. Math. 695 (2014), 175-214.

[29] J. Kulesza. Zero-dimensional covers of finite-dimensional dynamical systems. Ergodic Theory Dynam. Systems 15 (1995), 939-950.

[30] H. Lin and N. C. Phillips. Crossed products by minimal homeomorphisms. J. reine angew. Math. 641 (2010), 95-122.

[31] Q. Lin and N. C. Phillips. Direct limit decomposition for $\mathrm{C}^{*}$-algebras of minimal diffeomorphisms. In: Operator Algebras and Applications, pp. 107-133. Adv. Stud. Pure Math., 38, Math. Soc. Japan, Tokyo, 2004.

[32] E. Lindenstrauss. Mean dimension, small entropy factors and an embedding theorem. Inst. Hautes Études Sci. Publ. Math. 89 (1999), 227-262.

[33] E. Lindenstrauss and B. Weiss. Mean topological dimension. Israel J. Math. 115 (2000), 1-24.

[34] H. Matui. Homology and topological full groups of étale groupoids on totally disconnected spaces. Proc. Lond. Math. Soc. (3) 104 (2012), 27-56.

[35] H. Matui and Y. Sato. Strict comparison and $\mathscr{Z}$-absorption of nuclear $\mathrm{C}^{*}$-algebras. Acta Math. 209 (2012), 179-196.

[36] H. Matui and Y. Sato. Decomposition rank of UHF-absorbing C*-algebras. Duke Math. J. 163 (2014), 2687-2708.

[37] D. S. Ornstein and B. Weiss. Entropy and isomorphism theorems for actions of amenable groups. J. Anal. Math. 48 (1987), 1-141.

[38] N. C. Phillips. A classification theorem for nuclear purely infinite simple C*-algebras. Doc. Math. 5 (2000), 49-114.

[39] I. F. Putnam. On the topological stable rank of certain transformation group C*-algebras. Ergodic Theory Dynam. Systems 10 (1990), 197-207.

[40] M. Shub and B. Weiss. Can one always lower topological entropy? Ergodic Theory Dynam. Systems 11 (1991), 535-546.

[41] G. Szabó. The Rokhlin dimension of topological $\mathbb{Z}^{m}$-actions. Proc. Lond. Math. Soc. (3) 110 (2015), 673-694.

[42] G. Szabó, J. Zacharias, and J. Wu. Rokhlin dimension for actions of residually finite groups. Ergodic Theory Dynam. Systems (2017). DOI: 10.1017/etds.2017.113.

[43] A. Tikuisis, S. White, and W. Winter. Quasidiagonality of nuclear C*-algebras. Ann. of Math. (2) 185 (2017), 229-284.

[44] A. S. Toms and W. Winter. Minimal dynamics and K-theoretic rigidity: Elliott's conjecture. Geom. Funct. Anal. 23 (2013), 467-481.

[45] J.-L. Tu. La conjecture de Baum-Connes pour les feuilletages moyennables. K-Theory 17 (1999), 215264. 
[46] W. Winter. Nuclear dimension and $\mathscr{Z}$-stability of pure $\mathrm{C}^{*}$-algebras. Invent. Math. 187 (2012), 259-342.

David Kerr, Department of Mathematics, Mailstop 3368, Texas A\&M University, College STATION, TX 77843-3368, USA

E-mail address: kerr@math.tamu.edu

Gábor Szabó, Ku Leuven, Department of Mathematics, Celestijnenlaan 200B, Box 2400, B3001 Leuven, Belgium

E-mail address: gabor.szabo@kuleuven.be 